\title{
The Expressive Power of $k$-ary Exclusion Logic
}

\author{
Raine Rönnholm
}

Tampere University

\begin{abstract}
In this paper we study the expressive power of $k$-ary exclusion logic, $\operatorname{EXC}[k]$, that is obtained by extending first order logic with $k$-ary exclusion atoms. It is known that without arity bounds exclusion logic is equivalent with dependence logic. By observing the translations, we see that the expressive power of $\operatorname{EXC}[k]$ lies in between $k$-ary and $(k+1)$-ary dependence logics. We will show that, at least in the case when $k=1$, both of these inclusions are proper.

In a recent work by the author it was shown that $k$-ary inclusionexclusion logic is equivalent with $k$-ary existential second order logic, $\mathrm{ESO}[k]$. We will show that, on the level of sentences, it is possible to simulate inclusion atoms with exclusion atoms, and in this way express $\mathrm{ESO}[k]$-sentences by using only $k$-ary exclusion atoms. For this translation we also need to introduce a novel method for "unifying" the values of certain variables in a team. As a consequence, $\mathrm{EXC}[k]$ captures $\mathrm{ESO}[k]$ on the level of sentences, and we obtain a strict arity hierarchy for exclusion logic. It also follows that $k$-ary inclusion logic is strictly weaker than $\operatorname{EXC}[k]$. Finally we use similar techniques to formulate a translation from $\mathrm{ESO}[k]$ to $k$-ary inclusion logic with an alternative strict semantics. Consequently, for any arity fragment of inclusion logic, strict semantics is strictly more expressive than lax semantics.
\end{abstract}

Keywords: exclusion logic, inclusion logic, dependence logic, team semantics, existential second order logic, expressive power.

\section{Introduction}

Exclusion logic is an extension of first order logic with team semantics. In team semantics the truth of formulas is interpreted by using sets of assignments which are called teams. This approach was introduced by Hodges [14] to define compositional semantics for the IF-logic by Hintikka and Sandu [12]. The truth for the IF-logic was originally defined by using semantic games of imperfect information ([13]), and in thus teams can be seen as sets of parallel positions in a semantic game. Teams can also be interpreted as databases ([20]), and thus the study of logics with team semantics has natural connections with the study of database dependencies.

For first order logic team semantics is just a generalization of Tarski semantics and has the same expressive power. But if we extend first order logic 
with new atomic formulas, we obtain higher expressive power and we can define more complex properties of teams. The first new atoms for this framework were dependence atoms introduced by Väänänen [20]. In dependence logic the semantics for these atoms are defined by functional dependencies of the values of variables in a team. Several new atoms have been presented for this framework with the motivation from simple database dependencies - such as independence atoms by Grädel and Väänänen [8] and inclusion and exclusion atoms by Galliani [5]. Lately there has been research on these atoms with an attempt to formalize the dependency phenomena in different fields of science, such as database theory ([16]), belief presentation ([4]) and quantum mechanics ([15]).

If we extend first order logic with inclusion/exclusion atoms we obtain inclusion and exclusion logics. The team semantics for these atoms is very simple: Suppose that $\vec{t}_{1}, \vec{t}_{2}$ are $k$-tuples of terms and $X$ is a team. The $k$-ary inclusion atom $\overrightarrow{t_{1}} \subseteq \vec{t}_{2}$ says that the values of $\vec{t}_{1}$ are included in the values of $\vec{t}_{2}$ in the team $X$. The $k$-ary exclusion atom $\vec{t}_{1} \mid \vec{t}_{2}$ dually says that $\vec{t}_{1}$ and $\vec{t}_{2}$ get distinct values in $X$, i.e. for all assignments $s, s^{\prime} \in X$ we have $s\left(\vec{t}_{1}\right) \neq s^{\prime}\left(\vec{t}_{2}\right)$.

Galliani [5] has shown that without arity bounds exclusion logic is equivalent with dependence logic. Thus, on the level of sentences, it captures existential second order logic, ESO ([20]). Inclusion logic is not comparable with dependence logic in general ([5]), but captures positive greatest fixed point logic on the level of sentences, as shown by Galliani and Hella [7]. Hence exclusion logic captures NP and inclusion logic captures PTIME over finite structures with linear order.

In order to understand the nature of these atoms, there has been research on the bounded arity fragments of the corresponding logics. Durand and Kontinen [3] have shown that, on the level of sentences, $k$-ary dependence logic captures the fragment of ESO in which at most $(k-1)$-ary functions can be quantified. ${ }^{1}$ From this it follows that dependence logic has a strict arity hierarchy over sentences since the arity hierarchy of ESO (over arbitrary vocabulary) is known to be strict, as shown by Ajtai [1]. However, these earlier results do not tell much about the expressive power of $k$-ary exclusion logic, $\mathrm{EXC}[k]$, as the existing translation from it to dependence logic does not respect the arities of atoms.

There has not been much research on exclusion logic after Galliani proved its equivalence to dependence logic. In this paper we will show that the relationship between these two logics becomes nontrivial when we consider their bounded arity fragments. This also leads to results on the relation between inclusion and exclusion logics, which is interesting because they can be seen as duals to each other, as we have argued in [19].

By inspecting Galliani's translations ([5]) between exclusion and dependence logics more closely, we observe that $\mathrm{EXC}[k]$ is stronger than $k$-ary dependence logic but weaker than $(k+1)$-ary dependence logic. Thus it is natural to ask whether the expressive power of $\operatorname{EXC}[k]$ is strictly in between $k$-ary and $(k+1)$ ary dependence logics. We will show that this holds at least when $k=1$.

In an earlier work by the author [19] it was shown that both INC $[k]$ - and $\mathrm{EXC}[k]$-formulas could be translated into $k$-ary $\mathrm{ESO}, \mathrm{ESO}[k]$, which gives us an

\footnotetext{
${ }^{1}$ See [6], [9] and [10] for similar hierarchy results on independence and inclusion logics.
} 
upper bound for the expressive power of $\mathrm{EXC}[k]$. In [19] it was also shown that conversely $\mathrm{ESO}[k]$-formulas with at most $k$-ary free relation variables can be expressed in $k$-ary inclusion-exclusion logic, INEX $[k]$, and consequently INEX $[k]$ captures $\mathrm{ESO}[k]$ on the level of sentences.

Since exclusion logic is closed downwards, unlike inclusion-exclusion logic, we know that $\operatorname{EXC}[k]$ is strictly weaker than INEX $[k]$. However, in certain cases we can simulate the use of inclusion atoms with exclusion atoms: Suppose that $x, w, w^{c}$ are variables such that the sets of values of $w$ and $w^{c}$ in $X$ are complements of each other. Now we have $\mathcal{M} \vDash_{X} x \subseteq w$ iff $\mathcal{M} \vDash_{X} x \mid w^{c}$. This can be generalized for $k$-ary atoms if the values of $k$-tuples $\vec{w}$ and $\vec{w}^{c}$ are complementary (with respect to the full relation $M^{k}$ ).

We will use the observation above to modify our translation (in [19]) from $\mathrm{ESO}[k]$ to INEX $[k]$. If we only consider sentences of exclusion logic, we can quantify the needed complementary values, and then replace inclusion atoms in the translation with the corresponding exclusion atoms. The remaining problem is that in our translation we also needed a new connective called term value preserving disjunction ([19]) to avoid the loss of information on the values of certain variables when evaluating disjunctions. This operator can be defined by using both inclusion and exclusion atoms ([19]), but it is undefinable in exclusion logic since it is not closed downwards.

In [19] we have introduced new operators called inclusion and exclusion quantifiers and defined them in inclusion-exclusion logic. Furthermore, we have shown that universal inclusion quantifier $(\forall \vec{x} \subseteq \vec{t})$ could be defined also in exclusion logic. A natural reading for this quantifier is: "for all the values of $\vec{x}$ that are included in the values of $\vec{t}$ ". We will now consider the use of this quantifier in somewhat trivial looking form $(\forall \vec{x} \subseteq \vec{x})$. This operator turns out to be useful as it "unifies" the values of variables in a team. We will use it to define new operators called unifier, unified existential quantifier and unifying disjunction.

Unifying disjunction will give us an alternative method to avoid the loss of information in the translation from $\mathrm{ESO}[k]$. This completes our translation and proves the equivalence between $\mathrm{EXC}[k]$ and $\mathrm{ESO}[k]$ on the level of sentences. Hence we also obtain a strict arity hierarchy for exclusion logic since the arity hierarchy for ESO is known to be strict. We also get an interesting consequence that $k$-ary inclusion logic is strictly weaker than $\mathrm{EXC}[k]$ on the level of sentences (for any $k \geq 1$ ).

Finally, we will examine the expressive power of inclusion logic with an alternative semantics, so-called strict semantics. This semantical variant of inclusion logic has stronger expressive power by capturing the whole ESO, as shown by Galliani, Hannula and Kontinen [6], but lacks some nice semantical properties. We will use similar ideas, as in our translation from $\operatorname{ESO}[k]$ to $\mathrm{EXC}[k]$, to formulate a translation from $\mathrm{ESO}[k]$ to INC $[k]$ with strict semantics. Consequently, for any arity fragment of inclusion logic, strict semantics is more expressive than the standard semantics.

The structure of this paper is as follows: After preliminaries in Section 2, we present various new operators for exclusion logic Section 3 . In Section 4 we prove our main result by forming a translation from $\mathrm{ESO}[k]$ to $\mathrm{EXC}[k]$ on the 
level of sentences. Finally, in Section 5 , we present a translation from ESO $[k]$ to INC $[k]$ with strict semantics. This paper is an extended journal version of [18] with more detailed proofs and additional examples. Moreover, all the results in Section 5 are previously unpublished work.

\section{Preliminaries}

In this section we first present the team semantics for FO. Then we define inclusion and exclusion logics and review some of their known properties.

\subsection{Syntax and team semantics for FO}

A vocabulary $L$ is a set of relation symbols $R$, function symbols $f$ and constant symbols $c$. The set of $L$-terms, $\mathrm{T}_{L}$, is defined in the standard way. The set of variables occurring in a tuple $\vec{t}$ of $L$-terms is denoted by $\operatorname{Vr}(\vec{t})$.

Definition 2.1. The set of $\mathrm{FO}_{L}$-formulas is defined as follows:

$$
\varphi::=t_{1}=t_{2}\left|\neg t_{1}=t_{2}\right| R \vec{t}|\neg R \vec{t}|(\varphi \wedge \varphi)|(\varphi \vee \varphi)| \exists x \varphi \mid \forall x \varphi
$$

$\mathrm{FO}_{L}$-formulas of the form $t_{1}=t_{2}, \neg t_{1}=t_{2}, R \vec{t}$ and $\neg R \vec{t}$ are called literals.

Let $\varphi \in \mathrm{FO}_{L}$. We denote the set of subformulas of $\varphi$ by $\operatorname{Sf}(\varphi)$, the set of variables occurring in $\varphi$ by $\operatorname{Vr}(\varphi)$ and the set of free variables of $\varphi$ by $\operatorname{Fr}(\varphi)$.

An $L$-model $\mathcal{M}=(M, \mathcal{I})$, where the universe $M$ is any nonempty set and the interpretation $\mathcal{I}$ is a function whose domain is the vocabulary $L$. The interpretation $\mathcal{I}$ maps constant symbols to elements in $M, k$-ary relation symbols to $k$-ary relations in $M$ and $k$-ary function symbols to functions $M^{k} \rightarrow M$. For all $k \in L$ we write $k^{\mathcal{M}}:=\mathcal{I}(k)$. An assignment $s$ for $M$ is a function that is defined in some set of variables, $\operatorname{dom}(s)$, and ranges over $M$. A team $X$ for $M$ is any set of assignments for $M$ with a common domain, denoted by $\operatorname{dom}(X)$.

Let $s$ be an assignment and $a$ be any element in $M$. The assignment $s[a / x]$ is defined in $\operatorname{dom}(s) \cup\{x\}$, and it maps the variable $x$ to $a$ and all other variables as $s$. If $\vec{x}:=x_{1} \ldots x_{k}$ is a tuple of variables and $\vec{a}:=\left(a_{1}, \ldots, a_{k}\right) \in M^{k}$, we write $s[\vec{a} / \vec{x}]:=s\left[a_{1} / x_{1}, \ldots, a_{k} / x_{k}\right]$. For a team $X$, a set $A \subseteq M^{k}$ and for a function $\mathcal{F}: X \rightarrow \mathcal{P}\left(M^{k}\right) \backslash\{\emptyset\}$ we use the following notations.

$$
\left\{\begin{array}{l}
X[A / \vec{x}]:=\{s[\vec{a} / \vec{x}] \mid s \in X, \vec{a} \in A\} \\
X[\mathcal{F} / \vec{x}]:=\{s[\vec{a} / \vec{x}] \mid s \in X, \vec{a} \in \mathcal{F}(s)\} .
\end{array}\right.
$$

Let $\mathcal{M}$ be an $L$-model, $s$ an assignment and $t$ an $L$-term s.t. $\operatorname{Vr}(t) \subseteq \operatorname{dom}(s)$. The interpretation of $t$ with respect to $\mathcal{M}$ and $s$ is denoted simply by $s(t)$. For a team $X$ and a tuple $\vec{t}:=t_{1} \ldots t_{k}$ of $L$-terms we write

$$
s(\vec{t}):=\left(s\left(t_{1}\right), \ldots, s\left(t_{k}\right)\right) \text { and } X(\vec{t}):=\{s(\vec{t}) \mid s \in X\} .
$$

If $A \subseteq M^{k}$, we write $\bar{A}:=M^{k} \backslash A$. We are now ready to define team semantics for first order logic. 
Definition 2.2. Let $\mathcal{M}$ be an $L$-model, $\varphi \in \mathrm{FO}_{L}$ and $X$ a team such that $\operatorname{Fr}(\varphi) \subseteq \operatorname{dom}(X)$. We define the truth of $\varphi$ in the model $\mathcal{M}$ and the team $X$, $\mathcal{M} \vDash_{X} \varphi$, as follows.

- $\mathcal{M} \vDash_{X} t_{1}=t_{2}$ iff $s\left(t_{1}\right)=s\left(t_{2}\right)$ for all $s \in X$.

- $\mathcal{M} \vDash_{X} \neg t_{1}=t_{2}$ iff $s\left(t_{1}\right) \neq s\left(t_{2}\right)$ for all $s \in X$.

- $\mathcal{M} \vDash_{X} R \vec{t}$ iff $X(\vec{t}) \subseteq R^{\mathcal{M}}$.

- $\mathcal{M} \vDash_{X} \neg R \vec{t}$ iff $X(\vec{t}) \subseteq \overline{R^{\mathcal{M}}}$.

- $\mathcal{M} \vDash_{X} \psi \wedge \theta$ iff $\mathcal{M} \vDash_{X} \psi$ and $\mathcal{M} \vDash_{X} \theta$.

- $\mathcal{M} \vDash_{X} \psi \vee \theta$ iff there are $Y, Y^{\prime} \subseteq X$ s.t. $Y \cup Y^{\prime}=X, \mathcal{M} \vDash_{Y} \psi$ and $\mathcal{M} \vDash_{Y^{\prime}} \theta$.

- $\mathcal{M} \vDash_{X} \exists x \psi$ iff there exists $F: X \rightarrow \mathcal{P}(M) \backslash\{\emptyset\}$ s.t. $\mathcal{M} \vDash_{X[F / x]} \psi$.

- $\mathcal{M} \vDash_{X} \forall x \psi$ iff $\mathcal{M} \vDash_{X[M / x]} \psi$.

Remark. Above we have defined so-called lax-semantics in which we may select several witnesses when existentially quantifying variables and we may allow the "witnessing teams" ( $Y$ and $\left.Y^{\prime}\right)$ for disjunction to overlap. These operators also have an alternative so-called strict semantics:

- $\mathcal{M} \vDash_{X} \psi \vee \theta$ iff there are $Y, Y^{\prime} \subseteq X$ s.t. $Y \cup Y^{\prime}=X$, $Y \cap Y^{\prime}=\emptyset, \mathcal{M} \vDash_{Y} \psi$ and $\mathcal{M} \vDash_{Y^{\prime}} \theta$.

- $\mathcal{M} \vDash_{X} \exists x \psi$ iff there is $F: X \rightarrow M$ s.t. $\mathcal{M} \vDash_{X[F / x]} \psi$, where $X[F / x]$ is the team $\{x[F(s) / x] \mid s \in X\}$.

For FO these two semantic variants are equivalent. Galliani [5] has shown that they are also equivalent for exclusion logic but not for inclusion logic.

For tuples $\vec{t}:=t_{1} \ldots t_{k}$ and $\overrightarrow{t^{\prime}}:=t_{1}^{\prime} \ldots t_{k}^{\prime}$ of $L$-terms we write

$$
\vec{t}=\overrightarrow{t^{\prime}}:=\bigwedge_{i \leq k} t_{i}=t_{i}^{\prime} \quad \text { and } \quad \vec{t} \neq \overrightarrow{t^{\prime}}:=\bigvee_{i \leq k} \neg t_{i}=t_{i}^{\prime} .
$$

It is easy to see that the following equivalences hold:

$$
\begin{array}{ll}
\mathcal{M} \vDash_{X} \vec{t}=\overrightarrow{t^{\prime}} & \text { iff } s(\vec{t})=s\left(\overrightarrow{t^{\prime}}\right) \text { for all } s \in X \\
\mathcal{M} \vDash_{X} \vec{t} \neq \vec{t}^{\prime} & \text { iff } s(\vec{t}) \neq s\left(\overrightarrow{t^{\prime}}\right) \text { for all } s \in X .
\end{array}
$$

For $\varphi \in \mathrm{FO}_{L}$ and tuple $\vec{x}:=x_{1} \ldots x_{k}$ we write: $\exists \vec{x} \varphi:=\exists x_{1} \ldots \exists x_{k} \varphi$ and $\forall \vec{x} \varphi:=\forall x_{1} \ldots \forall x_{k} \varphi$. It is easy to show that

- $\mathcal{M} \vDash_{X} \exists \vec{x} \varphi$ iff there exists $\mathcal{F}: X \rightarrow \mathcal{P}\left(M^{k}\right) \backslash\{\emptyset\}$ s.t. $\mathcal{M} \vDash_{X[\mathcal{F} / \vec{x}]} \varphi$.

- $\mathcal{M} \vDash_{X} \forall \vec{x} \varphi$ iff $\mathcal{M} \vDash_{X\left[M^{k} / \vec{x}\right]} \varphi$.

In strict semantics the first condition turns into the form: $\mathcal{M} \vDash_{X} \exists \vec{x} \varphi$ iff there exists $\mathcal{F}: X \rightarrow M^{k}$ s.t. $\mathcal{M} \vDash_{X[\mathcal{F} / \vec{x}]} \varphi$, where $X[\mathcal{F} / \vec{x}]:=\{s[\mathcal{F}(s) / \vec{x}] \mid s \in X\}$. First order logic with team semantics has so-called flatness-property: 
Proposition 2.1 ([20], Flatness). Let $X$ be a team and $\varphi \in \mathrm{FO}_{L}$. The following equivalence holds: $\mathcal{M} \vDash_{X} \varphi$ iff $\mathcal{M} \vDash_{\{s\}} \varphi$ for all $s \in X$.

We use notations $\vDash_{s}^{\mathrm{T}}$ and $\vDash^{\mathrm{T}}$ for truth in a model with standard Tarski semantics. Team semantics can be seen just as a generalization of Tarski semantics as shown by the following proposition.

Proposition 2.2 ([20]). The following equivalences hold:

$$
\begin{array}{ll}
\mathcal{M} \vDash_{s}^{\mathrm{T}} \varphi \text { iff } \mathcal{M} \vDash_{\{s\}} \varphi & \text { for all } \mathrm{FO}_{L} \text {-formulas } \varphi \text { and assignments } s . \\
\mathcal{M} \vDash^{\mathrm{T}} \varphi \text { iff } \mathcal{M} \vDash_{\{\emptyset\}} \varphi & \text { for all } \mathrm{FO}_{L} \text {-sentences } \varphi .
\end{array}
$$

Note that, by flatness, $\mathcal{M} \vDash_{X} \varphi$ if and only if $\mathcal{M} \vDash_{s}^{\mathrm{T}} \varphi$ for all $s \in X$. By Proposition 2.2 it is natural to write $\mathcal{M} \vDash \varphi$, when we mean that $\mathcal{M} \vDash_{\{\emptyset\}} \varphi$. Note that $\mathcal{M} \vDash_{\emptyset} \varphi$ holds trivially for all $\mathrm{FO}_{L}$-formulas $\varphi$ by Definition 2.2. In general we say that any logic $\mathcal{L}$ with team semantics has the empty team property if $\mathcal{M} \vDash_{\emptyset} \varphi$ holds for all $\mathcal{L}$-formulas $\varphi$. We define three more important properties for any logic $\mathcal{L}$ with team semantics.

Definition 2.3. Let $\mathcal{L}$ be any logic with team semantics. We say that

- $\mathcal{L}$ is local, if the truth of formulas is determined only by the values of their free variables in a team, i.e. we have: $\mathcal{M} \vDash_{X} \varphi$ iff $\mathcal{M} \vDash_{X \mid \operatorname{Fr}(\varphi)} \varphi$.

- $\mathcal{L}$ is closed downwards if: $\mathcal{M} \vDash_{X} \varphi$ and $Y \subseteq X \Rightarrow \mathcal{M} \vDash_{Y} \varphi$.

- $\mathcal{L}$ is closed under unions if: $\mathcal{M} \vDash_{X_{i}} \varphi$ for every $i \in I \Rightarrow \mathcal{M} \vDash_{\cup_{i \in I} X_{i}} \varphi$.

By flatness it is easy to see that FO is local and closed both downwards and under unions.

\subsection{Inclusion and exclusion logics}

Inclusion logic (INC) and exclusion logic (EXC) are obtained by adding inclusion and exclusion atoms, respectively, to FO with team semantics.

Definition 2.4. If $\vec{t}_{1}, \vec{t}_{2}$ are $k$-tuples of $L$-terms, $\vec{t}_{1} \subseteq \vec{t}_{2}$ is a $k$-ary inclusion atom. $\mathrm{INC}_{L}$-formulas are formed like $\mathrm{FO}_{L}$-formulas by allowing the use of (non-negated) inclusion atoms like literals. Let $\mathcal{M}$ be a model and $X$ a team s.t. $\operatorname{Vr}\left(\vec{t}_{1} \vec{t}_{2}\right) \subseteq \operatorname{dom}(X)$. We define the truth of $\vec{t}_{1} \subseteq \vec{t}_{2}$ in $\mathcal{M}$ and $X$ as:

$$
\mathcal{M} \vDash_{X} \vec{t}_{1} \subseteq \overrightarrow{t_{2}} \text { iff for all } s \in X \text { there exists } s^{\prime} \in X \text { s.t. } s\left(\overrightarrow{t_{1}}\right)=s^{\prime}\left(\vec{t}_{2}\right) .
$$

Equivalently we have $\mathcal{M} \vDash_{X} \vec{t}_{1} \subseteq \vec{t}_{2}$ iff $X\left(\vec{t}_{1}\right) \subseteq X\left(\vec{t}_{2}\right)$.

If $\overrightarrow{t_{1}}, \overrightarrow{t_{2}}$ are $k$-tuples of $L$-terms, $\overrightarrow{t_{1}} \mid \overrightarrow{t_{2}}$ is a $k$-ary exclusion atom. $\mathrm{EXC}_{L^{-}}$ formulas are formed as $\mathrm{FO}_{L}$-formulas, but (non-negated) exclusion atoms may be used as literals are used in FO. Let $\mathcal{M}$ be a model and $X$ a team for which we have $\operatorname{Vr}\left(\vec{t}_{1} \vec{t}_{2}\right) \subseteq \operatorname{dom}(X)$. We define the truth of $\overrightarrow{t_{1}} \mid \overrightarrow{t_{2}}$ in $\mathcal{M}$ and $X$ as:

$$
\mathcal{M} \vDash_{X} \overrightarrow{t_{1}} \mid \overrightarrow{t_{2}} \text { iff for all } s, s^{\prime} \in X: s\left(\overrightarrow{t_{1}}\right) \neq s^{\prime}\left(\overrightarrow{t_{2}}\right) .
$$

Equivalently we have $\mathcal{M} \vDash_{X} \vec{t}_{1} \mid \vec{t}_{2}$ iff $X\left(\vec{t}_{1}\right) \cap X\left(\vec{t}_{2}\right)=\emptyset \quad\left(\right.$ iff $\left.X\left(\vec{t}_{1}\right) \subseteq \overline{X\left(\vec{t}_{2}\right)}\right)$. 
Inclusion-exclusion logic (INEX) is defined simply by allowing the use of both inclusion and exclusion atoms. If $\varphi \in \mathrm{EXC}_{L}$ contains at most $k$-ary exclusion atoms, we say that $\varphi$ is a formula of $k$-ary exclusion logic, $\operatorname{EXC}[k]$. Moreover, $k$-ary inclusion logic (INC $[k]$ ) and $k$-ary inclusion-exclusion logic $(\mathrm{INEX}[k])$ are defined analogously.

The following properties have all been shown by Galliani [5]: EXC, INC and INEX are all local and satisfy the empty team property. EXC is also closed downwards, unlike INC which is closed under unions. If we use strict semantics for INC, the resulting logic is not local. This is one of the reasons why the (lax)-semantics given in Definition 2.2 is usually considered to be more natural.

We denote inclusion logic with strict semantics by $\mathrm{INC}^{s}$ and its $k$-ary fragment by $\operatorname{INC}^{s}[k]$. Galliani, Hannula and Kontinen [6] have shown $\mathrm{INC}^{s}$ is equivalent with ESO. Thus, on the level of sentences, $\mathrm{INC}^{s}$ is equivalent with exclusion logic and stronger than (the standard) inclusion logic. We will study the properties of $\operatorname{INC}^{s}[k]$ in Section 5 .

\section{Useful operators for exclusion logic}

In this section we will define several operators for $\operatorname{EXC}[k]$. We first review how $k$-ary dependence atoms and intuitionistic disjunction can be expressed in $\mathrm{EXC}[k]$. Then we show how the values of certain tuples of terms can be unified by using universal inclusion quantifier that can be defined for EXC. With this technique we can define other useful operators for this framework.

\subsection{Dependence atoms and intuitionistic disjunction}

Let us review the semantics for dependence atoms of dependence logic ([20]). Let $t_{1} \ldots t_{k}$ be $L$-terms. The $k$-ary dependence atom $=\left(t_{1} \ldots t_{k-1}, t_{k}\right)$ has the following truth condition: $\mathcal{M} \vDash_{X}=\left(t_{1} \ldots t_{k-1}, t_{k}\right)$ if and only if we have:

for all $s, s^{\prime} \in X$ for which $s\left(t_{1} \ldots t_{k-1}\right)=s^{\prime}\left(t_{1} \ldots t_{k-1}\right)$ also $s\left(t_{k}\right)=s^{\prime}\left(t_{k}\right)$,

for all $L$-models $\mathcal{M}$ and teams $X$ for which $\operatorname{Vr}\left(t_{1} \ldots t_{k}\right) \subseteq \operatorname{dom}(X)$. This truth condition can be read as follows: "the value of $t_{k}$ is (functionally) dependent on the values of $t_{1}, \ldots, t_{k-1}$ ". By using Galliani's translation between dependence logic and exclusion logic, we can express $k$-ary dependence atoms in $\mathrm{EXC}[k]$ :

Proposition $3.1([5])$. Let $\vec{t}=t_{1} \ldots t_{k}$ be a tuple of L-terms. The k-ary dependence atom $=\left(t_{1} \ldots t_{k-1}, t_{k}\right)$ is equivalent with the $\mathrm{EXC}_{L}[k]$-formula $\varphi$ :

$$
\varphi:=\forall x\left(x=t_{k} \vee t_{1} \ldots t_{k-1} x \mid \vec{t}\right) \text {, where } x \text { is a fresh variable. }
$$

Hence, in particular, we can express constancy atom $]^{2}=(t)$ in $\operatorname{EXC}[k]$ for any $k \geq 1$. The semantics of intuitionistic disjunction $\sqcup$ is obtained by lifting the Tarski semantics of classical disjunction from single assignments to teams. That is, $\mathcal{M} \vDash_{X} \varphi \sqcup \psi$ iff $\mathcal{M} \vDash_{X} \varphi$ or $\mathcal{M} \vDash_{X} \psi$. Galliani [4] has shown that this operator can be expressed by using constancy atoms. Hence we can can define it as an abbreviation in $\mathrm{EXC}[k]$ for any $k \geq 1$.

\footnotetext{
${ }^{2}=(t)$ is true in a nonempty team $X$ iff $t$ has a constant value in $X$, i.e. $|X(t)|=1$.
} 


\subsection{Universal inclusion quantifier and unifier}

In [19] we have considered inclusion and exclusion dependencies from a new perspective by introducing inclusion and exclusion quantifiers. These quantifiers range over values of certain terms (or their complements) in the team instead of the whole universe $M$. We review here the semantics for universal inclusion and exclusion quantifiers $(\forall \vec{x} \subseteq \vec{t})$ and $(\forall \vec{x} \mid \vec{t})$. Let $\vec{x}$ be a $k$-tuple of variables, $\vec{t}$ a $k$-tuple of $L$-terms and $\varphi \in \operatorname{INEX}_{L}$. Now $(\forall \vec{x} \subseteq \vec{t})$ and $(\forall \vec{x} \mid \vec{t})$ have the following truth conditions:

$$
\begin{gathered}
\mathcal{M} \vDash_{X}(\forall \vec{x} \subseteq \vec{t}) \varphi \text { iff } \mathcal{M} \vDash_{X[A / \vec{x}]} \varphi, \text { where } A=X(\vec{t}) . \\
\mathcal{M} \vDash_{X}(\forall \vec{x} \mid \vec{t}) \varphi \text { iff } \mathcal{M} \vDash_{X[A / \vec{x}]} \varphi \text {, where } A=\overline{X(\vec{t})} .
\end{gathered}
$$

The quantifier $(\forall \vec{x} \subseteq \vec{t})$ has a natural reading: "for all tuples $\vec{x}$ that are included in the values of $\vec{t}$ ". And likewise $(\forall \vec{x} \mid \vec{t})$ can be read as: "for all tuples $\vec{x}$ that are excluded of the values of $\vec{t}$ ". These quantifiers can be defined in INEX by using the following idea: we first universally quantify $\vec{x}$ and then use inclusion and and exclusion atoms along with disjunction to force the team to be split into subteams $X[X(\vec{t}) / \vec{x}]$ and $X[\overline{X(\vec{t})} / \vec{x}]$; then we just state that $\varphi$ holds in the corresponding subteam (see [19] for more details).

In order to define these quantifiers as abbreviations in INEX we needed to use both $k$-ary inclusion and exclusion atoms (see [19] for details). However, we can alternatively define a quantifier $\left(\forall \vec{x} \subseteq^{e} \vec{t}\right)$ as an abbreviation by using only $k$-ary exclusion atoms ([19]). This quantifier has the same truth condition as $(\forall \vec{x} \subseteq \vec{t})$ above, when $\varphi$ is a formula of exclusion logic. Hence the universal inclusion quantifier for $k$-tuples of variables can be defined for both $\operatorname{INEX}[k]$ and $\mathrm{EXC}[k]$, although these definitions have to be given differently. From now on we will always use the plain notation $(\forall \vec{x} \subseteq \vec{t})$ and assume it be defined in the right way depending on whether we use it with INEX or EXC.

When defining quantifier $(\forall \vec{x} \subseteq \vec{t})$, we allowed the variables in the tuple $\vec{x}$ to occur in $\operatorname{Vr}(\vec{t})$. In particular, we accept the quantifiers of the form $(\forall \vec{x} \subseteq \vec{x})$. Quantifiers of this form may seem trivial, but they turn out to be rather useful operators. Let us analyze their truth condition:

$$
\mathcal{M} \vDash_{X}(\forall \vec{x} \subseteq \vec{x}) \varphi \text { iff } \mathcal{M} \vDash_{X^{\prime}} \varphi \text {, where } X^{\prime}=X[X(\vec{x}) / \vec{x}] .
$$

Note that the team $X^{\prime}$ is not necessarily the same team as $X$, although we have $\operatorname{dom}\left(X^{\prime}\right)=\operatorname{dom}(X)$ and even $X^{\prime}(\vec{x})=X(\vec{x})$. Consider the following example.

Example 3.1. Let $X=\left\{s_{1}, s_{2}\right\}$ where $s_{1}\left(v_{1}\right)=a, s_{2}\left(v_{1}\right)=b$ and $a \neq b$. Now

$$
\begin{aligned}
X\left[X\left(v_{1}\right) / v_{1}\right]=X\left[\{a, b\} / v_{1}\right] & =\left\{s_{1}\left[a / v_{1}\right], s_{1}\left[b / v_{1}\right], s_{2}\left[a / v_{1}\right], s_{2}\left[b / v_{1}\right]\right\} \\
& =\left\{s_{1}, s_{2}, s_{1}\left[b / v_{1}\right], s_{2}\left[a / v_{1}\right]\right\} .
\end{aligned}
$$

Thus, supposing that there is at least one variable $x \in \operatorname{dom}(X)$ for which $x \neq v_{1}$ and $s_{1}(x) \neq s_{2}(x)$, we have $X\left[X\left(v_{1}\right) / v_{1}\right] \neq X$.

We say that the quantifier $(\forall \vec{x} \subseteq \vec{x})$ unifies the values of the tuple $\vec{x}$ in a team. After executing this operation for a team $X$, then each assignment 
$s \in X \uparrow(\operatorname{dom}(X) \backslash \operatorname{Vr}(\vec{x}))$ "carries" the information on the whole relation $X(\vec{x})$. This also makes the values of the tuple $\vec{x}$ independent of all the other variables in $\operatorname{dom}(X)$. We can formulate this latter statement in independence logic $([8])$ as follows, when $\operatorname{Vr}(\vec{x}) \cap \operatorname{Vr}(\vec{v})=\emptyset$.

$\mathcal{M} \vDash_{X}(\forall \vec{x} \subseteq \vec{x}) \vec{x} \perp \vec{v}$ holds in any team $X$ for which $\operatorname{Vr}(\vec{v}) \subseteq \operatorname{dom}(X)$.

We introduce the following operator as an abbreviation.

Definition 3.1. Let $\vec{x}_{1}, \ldots, \vec{x}_{n}$ be tuples of variables and $\varphi \in \mathrm{EXC}_{L}$. The unifier of the values of $\vec{x}_{1}, \ldots, \vec{x}_{n}$, denoted by $\mathbf{U}\left(\vec{x}_{1}, \ldots, \vec{x}_{n}\right)$, is defined as:

$$
\mathbf{U}\left(\vec{x}_{1}, \ldots, \vec{x}_{n}\right) \varphi:=\left(\forall \vec{x}_{1} \subseteq \vec{x}_{1}\right) \ldots\left(\forall \vec{x}_{n} \subseteq \vec{x}_{n}\right) \varphi
$$

Note that tuples $\vec{x}_{1}, \ldots, \vec{x}_{n}$ above do not necessarily need to be of the same length. Moreover, they do not have to be disjoint, i.e the same variable may occur in more than one tuple. Also note that if the longest of the tuples $\vec{x}_{i}$ is a $k$-tuple, then this operator can be defined in $\operatorname{EXC}[k]$ (and in INEX $[k]$ ).

Example 3.2. We have $\mathbf{U}\left(\vec{x}_{1}, \ldots, \vec{x}_{n}\right) \varphi \equiv \mathbf{U}\left(\vec{x}_{1}\right) \ldots \mathbf{U}\left(\vec{x}_{n}\right) \varphi$ by the definition of the unifier. But one should note that usually

$$
\mathbf{U}\left(\vec{x}_{1} \ldots \vec{x}_{n}\right) \varphi \not \equiv \mathbf{U}\left(\vec{x}_{1}, \ldots, \vec{x}_{n}\right) \varphi .
$$

To see this, consider $X$ s.t. $v_{1}, v_{2} \in \operatorname{dom}(X)$ and let $X_{1}:=X\left[X\left(v_{1} v_{2}\right) / v_{1} v_{2}\right]$ and $X_{2}:=X\left[X\left(v_{1}\right) / v_{1}, X\left(v_{2}\right) / v_{2}\right]$. Now we have $X_{1}\left(v_{1} v_{2}\right)=X\left(v_{1} v_{2}\right)$ but $X_{2}\left(v_{1} v_{2}\right)=X\left(v_{1}\right) \times X\left(v_{2}\right)$. It is easy to see that $X_{1}$ and $X_{2}$ are identical only if $X\left(v_{1} v_{2}\right)=X\left(v_{1}\right) \times X\left(v_{2}\right)$.

We also note that the ordering of variables within the tuples does not effect the truth condition of the unifier. Hence for example $\mathbf{U}\left(x_{1} x_{2}\right) \varphi \equiv \mathbf{U}\left(x_{2} x_{1}\right) \varphi$ for any formula $\varphi$. Also clearly the repetitions of variables within the tuples do not matter, and thus for example $\mathbf{U}\left(x_{1} x_{1}\right) \varphi \equiv \mathbf{U}\left(x_{1}\right) \varphi$ for any $\varphi$.

The truth condition for the unifier is given by the following proposition whose truth is obvious.

Proposition 3.2. Let $\vec{x}_{1}, \ldots, \vec{x}_{n}$ be tuples of variables and $\varphi \in \mathrm{EXC}_{L}$. Now

$$
\mathcal{M} \vDash_{X} \mathbf{U}\left(\vec{x}_{1}, \ldots, \vec{x}_{n}\right) \varphi \text { iff } \mathcal{M} \vDash_{X\left[X\left(\vec{x}_{1}\right) / \vec{x}_{1}, \ldots, X\left(\vec{x}_{n}\right) / \vec{x}_{n}\right]} \varphi .
$$

When looking at Definition 3.1, it seems that if the tuples $\vec{x}_{1}, \ldots, \vec{x}_{n}$ are not disjoint, then their ordering affects the truth condition of $\mathbf{U}\left(\vec{x}_{1}, \ldots, \vec{x}_{n}\right)$. However, we can show that it is actually irrelevant in which order we unify the tuples. We first prove the following proposition which shows what happens when we unify two tuples which have some shared variables. The result shows that we obtain then the same result as when unifying separately the part that is overlapping and the disjoint parts.

Proposition 3.3. Let $x_{1}, \ldots, x_{k}$ be distinct variables and $1<m \leq n<k$. Then the following equivalence holds.

$$
\begin{aligned}
& \mathcal{M} \vDash_{X} \mathbf{U}\left(x_{1} \ldots x_{n}\right) \mathbf{U}\left(x_{m} \ldots x_{k}\right) \varphi \\
& \quad \text { iff } \mathcal{M} \vDash_{X} \mathbf{U}\left(x_{1} \ldots x_{m-1}\right) \mathbf{U}\left(x_{m} \ldots x_{n}\right) \mathbf{U}\left(x_{n+1} \ldots x_{k}\right) \varphi .
\end{aligned}
$$


Proof. We define the following teams:

$$
\begin{aligned}
& X_{1}::=X\left[X\left(x_{1} \ldots x_{n}\right) / x_{1} \ldots x_{n}\right] \\
& X_{2}:=X_{1}\left[X_{1}\left(x_{m} \ldots x_{k}\right) / x_{m} \ldots x_{k}\right] \\
& X_{3}:=X\left[X\left(x_{1} \ldots x_{m-1}\right) / x_{1} \ldots x_{m-1}, X\left(x_{m} \ldots x_{n}\right) / x_{m} \ldots x_{n},\right. \\
&\left.X\left(x_{n+1} \ldots x_{k}\right) / x_{n+1} \ldots x_{k}\right] .
\end{aligned}
$$

By the semantics of universal inclusion quantifier, it is sufficient to show that $X_{2}=X_{3}$.

For the sake of showing that $X_{3} \subseteq X_{2}$, let $s \in X_{3}$. Hence there is $r \in X$ and $a_{1} \ldots a_{m-1} \in X\left(x_{1} \ldots x_{m-1}\right), a_{m} \ldots a_{n} \in X\left(x_{m} \ldots x_{n}\right)$ and $a_{n+1} \ldots a_{k} \in$ $X\left(x_{n+1} \ldots x_{k}\right)$ such that

$$
s=r\left[a_{1} \ldots a_{m-1} / x_{1} \ldots x_{m-1}, a_{m} \ldots a_{n} / x_{m} \ldots x_{n}, a_{n+1} \ldots a_{k} / x_{n+1} \ldots x_{k}\right] .
$$

Moreover, there are $r_{1}, r_{2}, r_{3} \in X$ such that $r_{1}\left(x_{1}, \ldots x_{m-1}\right)=a_{1} \ldots a_{m-1}$, $r_{2}\left(x_{m}, \ldots x_{n}\right)=a_{m} \ldots a_{n}$ and $r_{3}\left(x_{n+1}, \ldots x_{k}\right)=a_{n+1} \ldots a_{k}$.

Let now $r^{\prime}:=r\left[r_{1}\left(x_{1} \ldots x_{n}\right) / x_{1} \ldots x_{n}\right]$ and $r^{\prime \prime}:=r_{3}\left[r_{2}\left(x_{1} \ldots x_{n}\right) / x_{1} \ldots x_{n}\right]$, whence $r^{\prime}, r^{\prime \prime} \in X_{1}$. It is quite easy to see that $s=r^{\prime}\left[r^{\prime \prime}\left(x_{m} \ldots x_{k}\right) / x_{m} \ldots x_{k}\right]$ and therefore $s \in X_{2}$.

For the sake of showing that $X_{2} \subseteq X_{3}$, let $s \in X_{2}$. Now there is $r_{1} \in X_{1}$ and $a_{m} \ldots a_{k} \in X_{1}\left(x_{m} \ldots x_{k}\right)$ such that $s=r_{1}\left[a_{m} \ldots a_{k} / x_{m} \ldots x_{k}\right]$. Moreover, there is $r_{2} \in X$ and $a_{1}^{\prime} \ldots a_{n}^{\prime} \in X\left(x_{1} \ldots x_{n}\right)$ such that $r_{1}=r_{2}\left[a_{1}^{\prime} \ldots a_{n}^{\prime} / x_{1} \ldots x_{n}\right]$. Since $a_{m} \ldots a_{k} \in X_{1}\left(x_{m} \ldots x_{k}\right)$, there is $r_{1}^{\prime} \in X_{1}$ s.t. $r_{1}^{\prime}\left(x_{m} \ldots x_{k}\right)=a_{m} \ldots a_{k}$. Furthermore there is $r_{2}^{\prime} \in X$ and a tuple $a_{1}^{\prime \prime} \ldots a_{n}^{\prime \prime} \in X\left(x_{1} \ldots x_{n}\right)$ such that $r_{1}^{\prime}=r_{2}^{\prime}\left[a_{1}^{\prime \prime} \ldots a_{n}^{\prime \prime} / x_{1} \ldots x_{n}\right]$. Let $a_{i}^{\prime \prime \prime}:=r_{2}^{\prime}\left(x_{i}\right)$ for each $i$ s.t. $n<i \leq k$. Now

$$
s=r_{2}\left[a_{1}^{\prime} \ldots a_{m-1}^{\prime} / x_{1} \ldots x_{m-1}, a_{m}^{\prime \prime} \ldots a_{n}^{\prime \prime} / x_{m} \ldots x_{n}, a_{n+1}^{\prime \prime \prime} \ldots a_{k}^{\prime \prime \prime} / x_{n+1} \ldots x_{k}\right] .
$$

Because we have $a_{1}^{\prime} \ldots a_{m-1}^{\prime} \in X\left(x_{1} \ldots x_{m-1}\right), a_{m}^{\prime \prime} \ldots a_{n}^{\prime \prime} \in X\left(x_{m} \ldots x_{n}\right)$ and $a_{n+1}^{\prime \prime \prime} \ldots a_{k}^{\prime \prime \prime} \in X\left(x_{n+1} \ldots x_{k}\right)$, it holds that $s \in X_{3}$.

Consider now some arbitrary tuples $\vec{x}_{1}$ and $\vec{x}_{2}$ of variables. Recalling the observations in Example 3.2, we can first rearrange these tuples in such a way that they match the assumptions of Proposition 3.3 and then unify the overlapping and disjoint parts separately. When unifying several tuples that are not disjoint, we can then show by a straightforward induction that one always obtains the same result by separately unifying some disjoint tuples. It then follows that the ordering of tuples $\vec{x}_{1}, \ldots, \vec{x}_{n}$ in $\mathbf{U}\left(\vec{x}_{1}, \ldots, \vec{x}_{n}\right)$ indeed does not affect its truth condition. For example we have

$$
\mathbf{U}\left(v_{1} v_{2}, v_{2} v_{3}\right) \varphi \equiv \mathbf{U}\left(v_{1}, v_{2}, v_{3}\right) \varphi \equiv \mathbf{U}\left(v_{3} v_{2}, v_{2} v_{1}\right) \varphi \equiv \mathbf{U}\left(v_{2} v_{3}, v_{1} v_{2}\right) \varphi .
$$

For the main results of this paper we only use the unifier for disjoint tuples and in this case the result of Proposition 3.3 is not needed. However, we think that this is an interesting property which could be useful when using unifier in some other context. 


\subsection{New operators that can be defined with unifier}

Unifier can be used in combination with other logical operators to form new useful tools for the framework of team semantics. We will introduce here two such operators. The definitions for the following operators are given more generally for INEX, but they can be defined in the same way for EXC as well.

Definition 3.2. Let $\vec{x}$ be a $k$-tuple of variables and $\varphi \in \operatorname{INEX}_{L}$. Unified existential quantifier $\exists^{\mathbf{U}}$ is defined as:

$$
\exists^{\mathbf{U}} \vec{x} \varphi:=\exists \vec{x} \mathbf{U}(\vec{x}) \varphi .
$$

Proposition 3.4. Let $\vec{x}$ be a $k$-tuple and $\varphi \in \operatorname{INEX}_{L}$. Now

$\mathcal{M} \vDash_{X} \exists^{\mathbf{U}} \vec{x} \varphi$ iff there exists a nonempty set $A \subseteq M^{k}$ s.t. $\mathcal{M} \vDash_{X[A / \vec{x}]} \varphi$.

Proof. If $X$ were the empty team, then the claim would hold trivially. Thus we may assume that $X \neq \emptyset$.

Suppose first that we have $\mathcal{M} \vDash_{X} \exists \mathbf{U} \vec{x} \varphi$, i.e. $\mathcal{M} \vDash_{X} \exists \vec{x} \mathbf{U}(\vec{x}) \varphi$. Therefore there exists a function $\mathcal{F}: X \rightarrow \mathcal{P}\left(M^{k}\right) \backslash\{\emptyset\}$ s.t. $\mathcal{M} \vDash_{X^{\prime}} \mathbf{U}(\vec{x}) \varphi$, where $X^{\prime}=X[\mathcal{F} / \vec{x}]$. Then $\mathcal{M} \vDash_{X^{\prime}\left[X^{\prime}(\vec{x}) / \vec{x}\right]} \varphi$. Since $X\left[X^{\prime}(\vec{x}) / \vec{x}\right]=X^{\prime}\left[X^{\prime}(\vec{x}) / \vec{x}\right]$ and $X^{\prime}(\vec{x}) \neq \emptyset$, we can choose $A:=X^{\prime}(\vec{x})$.

Suppose then that there exists nonempty $A \subseteq M^{k}$ s.t. $\mathcal{M} \vDash_{X[A / \vec{x}]} \varphi$. We define the function

$$
\mathcal{F}: X \rightarrow \mathcal{P}\left(M^{k}\right) \backslash\{\emptyset\}, \quad s \mapsto A \text { for all } s \in X .
$$

Let $X^{\prime}:=X[\mathcal{F} / \vec{x}]$, whence $X^{\prime}(\vec{x})=A$. Now $X^{\prime}\left[X^{\prime}(\vec{x}) / \vec{x}\right]=X^{\prime}[A / \vec{x}]=X[A / \vec{x}]$. Hence $\mathcal{M} \vDash_{X^{\prime}\left[X^{\prime}(\vec{x}) / \vec{x}\right]} \varphi$, and thus $\mathcal{M} \vDash_{X^{\prime}} \mathbf{U}(\vec{x}) \varphi$. Therefore $\mathcal{M} \vDash_{X} \exists \vec{x} \mathbf{U}(\vec{x}) \varphi$, i.e. $\mathcal{M} \vDash_{X} \exists^{\mathrm{U}} \vec{x} \varphi$.

If we use this quantifier in EXC (or in any other downwards closed logic), the following equivalence holds:

$$
\mathcal{M} \vDash_{X} \exists^{\mathbf{U}} \vec{x} \varphi \text { iff there exists } \vec{a} \in M^{k} \text { s.t. } \mathcal{M} \vDash_{X[\{\vec{a}\} / \vec{x}]} \varphi .
$$

For single variables this truth condition is equivalent with the semantics of the quantifier $\exists^{1}$ that was introduced in [17]. Note that in dependence logic this quantifier can be defined simply as $\exists^{1} x \varphi:=\exists x(=(x) \wedge \varphi)$.

The next operator will play a very important role in our translation from $\mathrm{ESO}[k]$ to $\mathrm{EXC}[k]$ in the next section.

Definition 3.3. Let $\varphi, \psi \in \operatorname{INEX}_{L}$ and let $\vec{x}_{1}, \ldots, \vec{x}_{n}$ be $k$-tuples of disjoint variables. Unifying disjunction for tuples $\vec{x}_{1}, \ldots, \vec{x}_{n}$ is defined as:

$$
\begin{array}{r}
\varphi_{\vec{x}_{1}, \ldots, \vec{x}_{n}} \vee:=\exists y_{1} \exists y_{2} \mathbf{U}\left(\vec{x}_{1}, \ldots, \vec{x}_{n}\right)\left(\left(y_{1}=y_{2} \wedge \varphi\right) \vee\left(y_{1} \neq y_{2} \wedge \psi\right)\right), \\
\text { where } y_{1}, y_{2} \text { are fresh variables. }
\end{array}
$$

Proposition 3.5. Let $\varphi, \psi \in \mathrm{INEX}_{L}$ and let $\vec{x}_{1}, \ldots, \vec{x}_{n}$ be $k$-tuples of variables. Now for all L-models $\mathcal{M}$ with at least two elements we have

$$
\begin{aligned}
& \mathcal{M} \vDash_{X} \varphi_{\vec{x}_{1}, \ldots, \vec{x}_{n}} \psi \vee \text { iff there exist } Y, Y^{\prime} \subseteq X \text { s.t. } Y \cup Y^{\prime}=X, \\
& \mathcal{M} \vDash_{Y\left[X\left(\vec{x}_{1}\right) / \vec{x}_{1}, \ldots, X\left(\vec{x}_{n}\right) / \vec{x}_{n}\right]} \varphi \text { and } \mathcal{M} \vDash_{Y^{\prime}\left[X\left(\vec{x}_{1}\right) / \vec{x}_{1}, \ldots, X\left(\vec{x}_{n}\right) / \vec{x}_{n}\right]} \psi .
\end{aligned}
$$


The intuitive idea about the proof of Proposition 3.5 is that before splitting the team, we must "announce" beforehand for each assignment if it will be placed on the left hand side or on the right hand side (or on both). This is done by giving the same or different values for the variables $y_{1}$ and $y_{2}$. Because the unification is done after this announcement, but before the actual splitting of the team, all the values will be unified correctly on both sides.

Proof. (Proposition 3.5) Because INEX is local, we may assume that $y_{1}, y_{2} \notin$ $\operatorname{dom}(X)$. Suppose first that $\mathcal{M} \vDash_{X} \varphi_{\vec{x}_{1}, \ldots, \vec{x}_{n}} \vee$, i.e.

$$
\mathcal{M} \vDash_{X} \exists y_{1} \exists y_{2} \mathbf{U}\left(\vec{x}_{1}, \ldots, \vec{x}_{n}\right)\left(\left(y_{1}=y_{2} \wedge \psi\right) \vee\left(y_{1} \neq y_{2} \wedge \theta\right)\right) .
$$

Thus there exist $F_{1}: X \rightarrow \mathcal{P}(M) \backslash\{\emptyset\}$ and $F_{2}: X\left[F_{1} / y_{1}\right] \rightarrow \mathcal{P}(M) \backslash\{\emptyset\}$ s.t.

$$
\mathcal{M} \vDash_{X_{1}} \mathbf{U}\left(\vec{x}_{1}, \ldots, \vec{x}_{n}\right)\left(\left(y_{1}=y_{2} \wedge \varphi\right) \vee\left(y_{1} \neq y_{2} \wedge \psi\right)\right) \text {, }
$$

where $X_{1}=X\left[F_{1} / y_{1}, F_{2} / y_{2}\right]$. Therefore $\mathcal{M} \vDash_{X_{2}}\left(y_{1}=y_{2} \wedge \varphi\right) \vee\left(y_{1} \neq y_{2} \wedge \psi\right)$, where $X_{2}=X_{1}\left[X_{1}\left(\vec{x}_{1}\right) / \vec{x}_{1}, \ldots, X_{1}\left(\vec{x}_{n}\right) / \vec{x}_{n}\right]$. Thus there exist $Z, Z^{\prime} \subseteq X_{2}$ s.t. $Z \cup Z^{\prime}=X_{2}, \mathcal{M} \vDash_{Z} y_{1}=y_{2} \wedge \varphi$ and $\mathcal{M} \vDash_{Z^{\prime}} y_{1} \neq y_{2} \wedge \psi$. Let

$$
\left\{\begin{array}{l}
Y:=\left\{s \in X \mid \text { There exists } a \in M \text { s.t. } s\left[a / y_{1}, a / y_{2}\right] \in X_{1}\right\} \\
Y^{\prime}:=\left\{s \in X \mid \text { There exist } a, b \in M \text { s.t. } a \neq b \text { and } s\left[a / y_{1}, b / y_{2}\right] \in X_{1}\right\} .
\end{array}\right.
$$

It is easy to see that $Y \cup Y^{\prime}=X$. Also note that since $X\left(\vec{x}_{i}\right)=X_{1}\left(\vec{x}_{i}\right)$ for each $i \leq n$, it holds that $X_{2}=X_{1}\left[X\left(\vec{x}_{1}\right) / \vec{x}_{1}, \ldots, X\left(\vec{x}_{n}\right) / x_{n}\right]$. We will show that $Y\left[X\left(\vec{x}_{1}\right) / \vec{x}_{1}, \ldots, X\left(\vec{x}_{n}\right) / \vec{x}_{n}\right]=Z\lceil\operatorname{dom}(X)$.

Let $r \in Y\left[X\left(\vec{x}_{1}\right) / \vec{x}_{1}, \ldots, X\left(\vec{x}_{n}\right) / \vec{x}_{n}\right]$. Now there exists $s \in Y$ and tuples $\vec{a}_{1} \in X\left(\vec{x}_{1}\right), \ldots, \vec{a}_{n} \in X\left(\vec{x}_{n}\right)$ s.t. $r=s\left[\vec{a}_{1} / \vec{x}_{1}, \ldots, \vec{a}_{n} / \vec{x}_{n}\right]$. Since $s \in Y$, there exists $a \in M$ s.t. $q:=s\left[a / y_{1}, a / y_{2}\right] \in X_{1}$. Let $q^{\prime}:=q\left[\vec{a}_{1} / \vec{x}_{1}, \ldots, \vec{a}_{n} / \vec{x}_{n}\right]$, whence $q^{\prime} \in X_{2}$. Since $q^{\prime}\left(y_{1}\right)=a=q^{\prime}\left(y_{2}\right)$ and $\mathcal{M} \vDash_{Z^{\prime}} y_{1} \neq y_{2}$ we have $q^{\prime} \notin Z^{\prime}$, and thus it must be that $q^{\prime} \in Z$. But since now $r=q^{\prime} \uparrow \operatorname{dom}(X) \in Z \uparrow \operatorname{dom}(X)$, we have shown that $Y\left[X\left(\vec{x}_{1}\right) / \vec{x}_{1}, \ldots, X\left(\vec{x}_{n}\right) / \vec{x}_{n}\right] \subseteq Z \uparrow \operatorname{dom}(X)$.

Let then $r^{*} \in Z\left\lceil\operatorname{dom}(X)\right.$. Now there exists $r \in Z$ s.t. $r^{*}=r \uparrow \operatorname{dom}(X)$. Because $\mathcal{M} \vDash_{Z} y_{1}=y_{2}$ it must be that $r\left(y_{1}\right)=r\left(y_{2}\right)$. Since $r \in Z \subseteq X_{2}$ there exists $q \in X_{1}$ and $\vec{a}_{1} \in X\left(\vec{x}_{1}\right), \ldots, \vec{a}_{n} \in X\left(\vec{x}_{n}\right)$ s.t. $r=q\left[\vec{a}_{1} / \vec{x}_{1}, \ldots, \vec{a}_{n} / \vec{x}_{n}\right]$. Let $s:=q \uparrow \operatorname{dom}(X)$. Since $q\left(y_{1}\right)=r\left(y_{1}\right)=r\left(y_{2}\right)=q\left(y_{2}\right)$ and $s \in X$, by the definition of $Y$ we have $s \in Y$. Let $s^{\prime}:=s\left[\vec{a}_{1} / \vec{x}_{1}, \ldots, \vec{a}_{n} / \vec{x}_{n}\right]$, whence $s^{\prime} \in Y\left[X\left(\vec{x}_{1}\right) / \vec{x}_{1}, \ldots, X\left(\vec{x}_{n}\right) / \vec{x}_{n}\right]$. But now it must also be that $s^{\prime}=r^{*}$ and thus $Z\left\lceil\operatorname{dom}(X) \subseteq Y\left[X\left(\vec{x}_{1}\right) / \vec{x}_{1}, \ldots, X\left(\vec{x}_{n}\right) / \vec{x}_{n}\right]\right.$.

We have shown that $Y\left[X\left(\vec{x}_{1}\right) / \vec{x}_{1}, \ldots, X\left(\vec{x}_{n}\right) / \vec{x}_{n}\right]=Z \uparrow \operatorname{dom}(X)$. Since $\mathcal{M} \vDash_{Z} \varphi$, by locality $\mathcal{M} \vDash_{Z\lceil\operatorname{dom}(X)} \varphi$ and thus $\mathcal{M} \vDash_{Y\left[X\left(\vec{x}_{1}\right) / \vec{x}_{1}, \ldots, X\left(\vec{x}_{n}\right) / \vec{x}_{n}\right]} \varphi$. With a similar argumentation $Y^{\prime}\left[X\left(\vec{x}_{1}\right) / \vec{x}_{1}, \ldots, X\left(\vec{x}_{n}\right) / \vec{x}_{n}\right]=Z^{\prime} \uparrow \operatorname{dom}(X)$ and consequently $\mathcal{M} \vDash_{Y^{\prime}\left[X\left(\vec{x}_{1}\right) / \vec{x}_{1}, \ldots, X\left(\vec{x}_{n}\right) / \vec{x}_{n}\right]} \psi$.

Suppose then that there are subteams $Y, Y^{\prime} \subseteq X$ such that $Y \cup Y^{\prime}=X$, $\mathcal{M} \vDash_{Y\left[X\left(\vec{x}_{1}\right) / \vec{x}_{1}, \ldots, X\left(\vec{x}_{n}\right) / \vec{x}_{n}\right]} \varphi$ and $\mathcal{M} \vDash_{Y^{\prime}\left[X\left(\vec{x}_{1}\right) / \vec{x}_{1}, \ldots, X\left(\vec{x}_{n}\right) / \vec{x}_{n}\right]} \psi$. Since $|M| \geq 2$, there 
exist $a, b \in M$ s.t. $a \neq b$. We define the following functions:

$$
\begin{aligned}
& F_{1}: X \rightarrow \mathcal{P}(M) \backslash\{\emptyset\}, \quad \begin{cases}s \mapsto\{a\} \quad \text { if } s \in Y \backslash Y^{\prime} \\
s \mapsto\{b\} \quad \text { if } s \in Y^{\prime} \backslash Y \\
s \mapsto\{a, b\} \text { if } s \in Y \cap Y^{\prime}\end{cases} \\
& F_{2}: X\left[F_{1} / y_{1}\right] \rightarrow \mathcal{P}(M) \backslash\{\emptyset\}, \quad s \mapsto\{a\} .
\end{aligned}
$$

We define teams $X_{1}:=X\left[F_{1} / y_{1}, F_{2} / y_{2}\right], X_{2}:=X_{1}\left[X\left(\vec{x}_{1}\right) / \vec{x}_{1}, \ldots, X\left(\vec{x}_{n}\right) / x_{n}\right]$, $Z:=\left\{s \in X_{2} \mid s\left(y_{1}\right)=s\left(y_{2}\right)\right\}$ and $Z^{\prime}:=\left\{s \in X_{2} \mid s\left(y_{1}\right) \neq s\left(y_{2}\right)\right\}$. Clearly now $Z \cup Z^{\prime}=X_{2}, \mathcal{M} \vDash_{Z} y_{1}=y_{2}$ and $\mathcal{M} \vDash_{Z^{\prime}} y_{1} \neq y_{2}$. We will then show that $Y\left[X\left(\vec{x}_{1}\right) / \vec{x}_{1}, \ldots, X\left(\vec{x}_{n}\right) / \vec{x}_{n}\right]=Z\lceil\operatorname{dom}(X)$.

Let $r \in Y\left[X\left(\vec{x}_{1}\right) / \vec{x}_{1}, \ldots, X\left(\vec{x}_{n}\right) / \vec{x}_{n}\right]$. Now there is $s \in Y$ and tuples $\vec{a}_{1} \in$ $X\left(\vec{x}_{1}\right), \ldots, \vec{a}_{n} \in X\left(\vec{x}_{n}\right)$ s.t. $r=s\left[\vec{a}_{1} / \vec{x}_{1}, \ldots, \vec{a}_{n} / \vec{x}_{n}\right]$. Since $s \in Y$, by the definition of $F_{1}$, we have $s\left[a / y_{1}\right] \in X\left[F_{1} / y_{1}\right]$. Let then $q:=s\left[a / y_{1}, a / y_{2}\right]$ and $q^{\prime}:=q\left[\vec{a}_{1} / \vec{x}_{1}, \ldots, \vec{a}_{n} / \vec{x}_{n}\right]$, whence $q \in X_{1}$ and $q^{\prime} \in X_{2}$. Since $q^{\prime}\left(y_{1}\right)=q^{\prime}\left(y_{2}\right)$, by the definition of $Z$, we have $q^{\prime} \in Z$. But now $r=q^{\prime} \uparrow \operatorname{dom}(X) \in Z \uparrow \operatorname{dom}(X)$, and thus we have shown that $Y\left[X\left(\vec{x}_{1}\right) / \vec{x}_{1}, \ldots, X\left(\vec{x}_{n}\right) / \vec{x}_{n}\right] \subseteq Z \uparrow \operatorname{dom}(X)$.

Let then $r^{*} \in Z \uparrow \operatorname{dom}(X)$. Now there is $r \in Z$ s.t. $r^{*}=r \uparrow \operatorname{dom}(X)$. By the definition of $Z$ we have $r\left(y_{1}\right)=r\left(y_{2}\right)$. Since $r \in Z \subseteq X_{2}$, there is $q \in X_{1}$ and tuples $\vec{a}_{1} \in X\left(\vec{x}_{1}\right), \ldots, \vec{a}_{n} \in X\left(\vec{x}_{n}\right)$ such that $r=q\left[\vec{a}_{1} / \vec{x}_{1}, \ldots, \vec{a}_{n} / \vec{x}_{n}\right]$. Let $s:=q\left\lceil\operatorname{dom}(X)\right.$. Since $q\left(y_{1}\right)=q\left(y_{2}\right)$, by the definition of $F_{1}$, we must have $s \in Y$. Let $s^{\prime}:=s\left[\vec{a}_{1} / \vec{x}_{1}, \ldots, \vec{a}_{n} / \vec{x}_{n}\right]$, whence $s^{\prime} \in Y\left[X\left(\vec{x}_{1}\right) / \vec{x}_{1}, \ldots, X\left(\vec{x}_{n}\right) / \vec{x}_{n}\right]$. But now $s^{\prime}=r^{*}$ and thus $Z\left\lceil\operatorname{dom}(X) \subseteq Y\left[X\left(\vec{x}_{1}\right) / \vec{x}_{1}, \ldots, X\left(\vec{x}_{n}\right) / \vec{x}_{n}\right]\right.$.

We have shown that $Y\left[X\left(\vec{x}_{1}\right) / \vec{x}_{1}, \ldots, X\left(\vec{x}_{n}\right) / \vec{x}_{n}\right]=Z \uparrow \operatorname{dom}(X)$. Thus by the initial assumption we have $\mathcal{M} \vDash_{Z\lceil\operatorname{dom}(X)} \varphi$ and thus by locality $\mathcal{M} \vDash_{Z} \varphi$. With similar argumentation we can show that $Y^{\prime}\left[X\left(\vec{x}_{1}\right) / \vec{x}_{1}, \ldots, X\left(\vec{x}_{n}\right) / \vec{x}_{n}\right]=$ $Z^{\prime}\left\lceil\operatorname{dom}(X)\right.$ and consequently $\mathcal{M} \vDash_{Z^{\prime}} \psi$.

Therefore it holds that $\mathcal{M} \vDash_{Z} y_{1}=y_{2} \wedge \varphi$ and $\mathcal{M} \vDash_{Z^{\prime}} y_{1} \neq y_{2} \wedge \psi$. Furthermore we can conclude that $\mathcal{M} \vDash_{X} \exists y_{1} \exists y_{2} \mathbf{U}\left(\vec{x}_{1}, \ldots, \vec{x}_{n}\right)\left(\left(y_{1}=y_{2} \wedge \psi\right) \vee\left(y_{1} \neq y_{2} \wedge \theta\right)\right)$, i.e. $\mathcal{M} \vDash_{X} \varphi_{\vec{x}_{1}, \ldots, \vec{x}_{n}} \vee^{\mathrm{U}} \psi$.

Remark. We could easily modify the definition of unifying disjunction to make it work properly also in the case of single element models. Let

$$
\varphi_{\vec{x}_{1}, \ldots, \vec{x}_{n}} \vee^{\mathbf{U}} \psi:=\left(\forall z_{1} \forall z_{2}\left(z_{1}=z_{2}\right) \wedge(\varphi \vee \psi)\right) \sqcup \varphi_{\vec{x}_{1}, \ldots, \vec{x}_{n}} \vee^{\mathbf{U}} \psi
$$

It is easy to see that the truth condition given by Proposition 3.5 holds for the operator above even without the extra assumption $|M|>1$, as unifying disjunction becomes normal disjunction in the case of single element models. However, in this paper we are mainly using this operator as a tool in our main translation (Theorem 4.5) where this simpler form suffices for our needs.

\section{The expressive power of $\mathrm{EXC}[k]$}

In this section we analyze the expressive power of $\mathrm{EXC}[k]$ by comparing it with $k$-ary dependence logic and $k$-ary existential second order logic. Finally we discuss the correspondence between $\mathrm{EXC}[k]$ and INC $[k]$. 
Since the lax- and strict semantics are equivalent for exclusion logic, we may freely use either of them. In order to simplify some proofs in this section we decide to use the strict semantics for existential quantifier and lax-semantics for disjunction 3

\subsection{Relationship between EXC and dependence logic}

Galliani [5] has shown that, without arity bounds, EXC is equivalent with dependence logic. However, if we consider the bounded arity fragments, this relationship becomes nontrivial. We first review Galliani's translation from exclusion logic to dependence logic (the translation is slightly simplified here).

Proposition 4.1 ([5]). Let $\vec{t}_{1}, \vec{t}_{2}$ be $k$-tuples of L-terms. The $k$-ary exclusion atom $\overrightarrow{t_{1}} \mid \overrightarrow{t_{2}}$ is logically equivalent to the depencende logic formula $\varphi$ :

$\varphi:=\forall \vec{y} \exists w_{1} \exists w_{2}\left(=\left(w_{1}\right) \wedge=\left(\vec{y}, w_{2}\right) \wedge\left(\left(w_{1}=w_{2} \wedge \vec{y} \neq \vec{t}_{1}\right) \vee\left(w_{1} \neq w_{2} \wedge \vec{y} \neq \overrightarrow{t_{2}}\right)\right)\right)$, where $\vec{y}$ is a $k$-tuple of fresh variables and $w_{1}, w_{2}$ are fresh variables.

By inspecting Galliani's translations, we obtain the following result on the relationship between the arity fragments of exclusion logic and dependence logic.

Corollary 4.2. The expressive power of $\mathrm{EXC}[k]$ is in between $k$-ary dependence logic and $(k+1)$-ary dependence logic on the level of formulas.

Proof. By using the translation in Proposition 3.1 we can express $k$-ary dependence atoms with $k$-ary exclusion atoms. Moreover, by using the translation in Proposition 4.1 we can express $k$-ary exclusion atoms with $(k+1)$-ary dependence atoms.

By this result it is natural to ask whether these inclusions are proper, or whether $\mathrm{EXC}[k+1]$ collapses to some arity fragment of dependence logic. Let us inspect the special case $k=1$ with the following example.

Example 4.1 (C.f. a similar example for INEX in [19]). Let $\mathcal{G}=(V, E)$ be an undirected graph. Now we have

(a) $\mathcal{G}$ is disconnected if and only if

$$
\mathcal{G} \vDash \forall z \exists x_{1} \exists x_{2}\left(\left(x_{1}=z \vee x_{2}=z\right) \wedge x_{1} \mid x_{2} \wedge\left(\forall y_{1} \subseteq x_{1}\right)\left(\forall y_{2} \subseteq x_{2}\right) \neg E y_{1} y_{2}\right) .
$$

(b) $\mathcal{G}$ is $k$-colorable if and only if

$$
\begin{aligned}
\mathcal{G} \vDash \gamma_{\leq k} \sqcup \forall z \exists x_{1} \ldots \exists x_{k}\left(\bigvee_{i \leq k} x_{i}\right. & =z \wedge \bigwedge_{i \neq j} x_{i} \mid x_{j} \\
& \left.\wedge \bigwedge_{i \leq k}\left(\forall y_{1} \subseteq x_{i}\right)\left(\forall y_{2} \subseteq x_{i}\right) \neg E y_{1} y_{2}\right),
\end{aligned}
$$

where $\gamma_{\leq k}:=\exists x_{1} \ldots \exists x_{k} \forall y\left(\bigvee_{i \leq k} y=x_{i}\right)$.

\footnotetext{
${ }^{3}$ This combination is in some sense the simplest choice. It was used originally when dependence logic was defined ([20]). The lax- and strict-separation was noticed only after introducing logics that were not closed downwards.
} 
We explain briefly why these equivalences hold. In (a), suppose that the given sentence is true in $\mathcal{G}$. Let $X$ be the team after the quantification of $z, x_{1}$ and $x_{2}$. Since we have $\mathcal{M} \vDash_{X} x_{1}=z \vee x_{2}=z$ and $X(z)=V$, it must be that $X\left(x_{1}\right) \cup X\left(x_{2}\right)=V$. And since $\mathcal{M} \vDash_{X} x_{1} \mid x_{2}$, it must be that $X\left(x_{1}\right) \cap X\left(x_{2}\right)=\emptyset$. Hence the sets $X\left(x_{1}\right)$ and $X\left(x_{2}\right)$ must form a disjoint union of all vertices. Because $\mathcal{M} \vDash_{X}\left(\forall y_{1} \subseteq x_{1}\right)\left(\forall y_{2} \subseteq x_{2}\right) \neg E y_{1} y_{2}$, we have $(a, b) \notin E$ for any pair $(a, b)$ in $X\left(x_{1}\right) \times X\left(x_{2}\right)$. That is, there is no edge between these disjoint sets and thus $\mathcal{G}$ must be disconnected. It is easy to see that also the converse claim holds.

Let us then consider the equivalence in (b). If $\mathcal{G} \vDash \gamma_{\leq k}$ the graph is trivially $k$-colorable. Else let $X$ be the team after the quantification of variables $z, x_{1}, \ldots, x_{k}$. As above, the truth of $\bigvee_{i \leq k} x_{i}=z$ guarantees that $\bigcup_{i \leq k} X\left(x_{i}\right)=V$ and the truth of exclusion atoms guarantees that sets $X\left(x_{i}\right)$ are disjoint. Let these sets be the coloring of the graph. Because we have for all $i \leq n$ : $\mathcal{M} \vDash_{X}\left(\forall y_{1} \subseteq x_{i}\right)\left(\forall y_{2} \subseteq x_{i}\right) \neg E y_{1} y_{2}$, it follows that $(a, b) \notin E$ for any pair $a, b \in X_{i}$ and $i \leq n$. That is, there is no edge between any two vertices chosen from a single color set, i.e. the coloring is correct. It is easy to see that also the converse claim holds.

Corollary 4.3. The expressive power of $\mathrm{EXC}[1]$ is properly in between 1-ary and 2-ary dependence logics, on the level of both sentences and formulas.

Proof. By Corollary 4.2, the expressive power of EXC[1] is in between 1-ary and 2-ary dependence logics. By the results of Galliani [5], 1-ary dependence logic is not stronger than FO on the level of sentences. However, by Example 4.1, there are sentences of EXC[1] that cannot be expressed in FO. Thus EXC[1] is strictly stronger than 1-ary dependence logic on the level of sentences.

On the other hand, there are properties that are definable 2-ary dependence logic, but which cannot be expressed in existential monadic second order logic, EMSO, such as infinity of a model and even cardinality ([20]). But since INEX[1] is equivalent to EMSO on the level of sentences ([19]), EXC[1] must be strictly weaker than 2-ary dependence logic on the level of sentences.

\subsection{Capturing the arity fragments of ESO with EXC}

In this subsection we will compare the expressive power of EXC with existential second order logic, ESO. We denote the $k$-ary fragment of ESO (where at most $k$-ary relation symbols can be quantified) by $\mathrm{ESO}[k]$. We will formulate a translation from $\mathrm{ESO}[k]$ to $\mathrm{EXC}[k]$ on the level of sentences by using the idea from the following observation: Suppose that $X$ is a team and $\vec{x}, \vec{w}, \vec{w}^{c}$ are tuples variables s.t. $X\left(\vec{w}^{c}\right)=\overline{X(\vec{w})}$. Now $\mathcal{M} \vDash_{X} \vec{x} \subseteq \vec{w}$ iff $\mathcal{M} \vDash_{X} \vec{x} \mid \vec{w}^{c}$.

In our translation from $\mathrm{ESO}[k]$ to $\operatorname{INEX}[k]([19])$ the quantified $k$-ary relation symbols $P_{i}$ of an $\mathrm{ESO}_{L}$-formula were simply replaced with $k$-tuples $\vec{w}_{i}$ of quantified first order variables. Then the formulas of the form $P_{i} \vec{t}$ were replaced with the inclusion atoms $\vec{t} \subseteq \vec{w}_{i}$ and the formulas of the form $\neg P_{i} \vec{t}$ with the exclusion atoms $\vec{t} \mid \vec{w}_{i}$. In order to eliminate inclusion atoms from this translation, we also need to quantify a tuple $\vec{w}_{i}^{c}$ of variables for each $P_{i}$ and set a requirement that $\vec{w}_{i}^{c}$ must be given complementary values with respect to the values of $\vec{w}_{i}$. This requirement is possible to be set in exclusion logic if we are 
restricted to sentences. Then we simply replace inclusion atoms $\vec{t} \subseteq \vec{w}_{i}$ with the corresponding exclusion atoms $\vec{t} \mid \vec{w}_{i}^{c}$.

We also need to consider the quantification of the empty set and the full relation $M^{k}$ as special cases. This is because tuples $\vec{w}_{i}$ and also their "complements" $\vec{w}_{i}^{c}$ must always be given a nonempty set of values. For this we use special "label variables" $w_{i}^{\circ}$ and $w_{i}^{\bullet}$ for each relation symbol $P_{i}$. We first quantify some constant value for a variable $u$. Then we can give the value of $u$ for $w_{i}^{\circ}$ to "announce" the quantification of the empty set or analogously we can give it for $w_{i}^{\bullet}$ to announce the quantification of the full relation. In order to give these label values, there must be at least two elements in the model. For handling the special case of single element models we will use the following easy lemma (we omit the proof).

Lemma 4.4. Let $\varphi$ be an $\mathrm{ESO}_{L}$-sentence. Now there exists an $\mathrm{FO}_{L}$-sentence $\chi$, such that we have $\mathcal{M} \vDash \varphi$ iff $\mathcal{M} \vDash \chi$, for all L-models $\mathcal{M}=(M, \mathcal{I})$ for which $|M|=1$.

The remaining problem is that in the translation from ESO to INEX we also needed a new connective called term value preserving disjunction ([19]) to avoid the "loss of information" on the values of variables $\vec{w}_{i}$ when evaluating disjunctions (as after splitting the team, there might be less values for some variables in the subteams). This time we can use unifying disjunction instead to avoid the loss of information on the values of both the tuples $\vec{w}_{i}$ and the tuples $\vec{w}_{i}^{c}$. We are now ready to formulate our main theorem.

Theorem 4.5. For every $\mathrm{ESO}_{L}[k]$-sentence $\Phi$ there exists an $\mathrm{EXC}_{L}[k]$-sentence $\varphi$ such that

$$
\mathcal{M} \vDash \varphi \quad \text { iff } \mathcal{M} \vDash \Phi .
$$

Proof. Since $\Phi$ is an $\mathrm{ESO}_{L}[k]$-sentence, there exists a $\mathrm{FO}_{L}$-sentence $\delta$ and relation symbols $P_{1}, \ldots, P_{n}$ so that $\Phi=\exists P_{1} \ldots \exists P_{n} \delta$. Without losing generality, we may assume that $P_{1}, \ldots, P_{n}$ are all $k$-ary. Let $\vec{w}_{1}, \ldots, \vec{w}_{n}$ and $\vec{w}_{1}^{c}, \ldots, \vec{w}_{n}^{c}$ be $k$-tuples of variables and $w_{1}^{\circ}, \ldots, w_{n}^{\circ}, w_{1}^{\bullet}, \ldots, w_{n}^{\bullet}$ and $u$ be variables such that all of these variables are distinct and do not occur in the sentence $\delta$.

Let $\psi \in \operatorname{Sf}(\delta)$. The formula $\psi^{\prime}$ is defined recursively:

$$
\begin{aligned}
\psi^{\prime} & =\psi \quad \text { if } \psi \text { is a literal and } P_{i} \text { does not occur in } \psi \text { for any } i \leq n \\
\left(P_{i} \vec{t}\right)^{\prime} & =\left(\vec{t} \mid \vec{w}_{i}^{c} \vee w_{i}^{\bullet}=u\right) \wedge w_{i}^{\circ} \neq u \quad \text { for all } i \leq n \\
\left(\neg P_{i} \vec{t}\right)^{\prime} & =\left(\vec{t} \mid \vec{w}_{i} \vee w_{i}^{\circ}=u\right) \wedge w_{i}^{\bullet} \neq u \quad \text { for all } i \leq n \\
(\psi \wedge \theta)^{\prime} & =\psi^{\prime} \wedge \theta^{\prime} \\
(\psi \vee \theta)^{\prime} & =\psi^{\prime} \underline{\vee}^{\mathbf{U}} \theta^{\prime}, \quad \text { where } \underline{\vee}^{\mathbf{U}}:=\underset{\vec{w}_{1}, \ldots, \vec{w}_{n}, \vec{w}_{1}^{c}, \ldots, \vec{w}_{n}^{c}}{\mathbf{U}^{c}} \\
(\exists x \psi)^{\prime} & =\exists x \psi^{\prime} \\
(\forall x \psi)^{\prime} & =\forall x \psi^{\prime} .
\end{aligned}
$$

Let $\chi$ be a $\mathrm{FO}_{L}$-sentence determined by the Lemma 4.4 for the sentence $\Phi$ and let $\vec{z}$ be a $k$-tuple of fresh variables. Let $\gamma_{=1}$ be an abbreviation for the 
sentence $\forall z_{1} \forall z_{2}\left(z_{1}=z_{2}\right)$. Now we can define the sentence $\varphi$ in the following way:

$$
\begin{aligned}
\varphi:=\left(\gamma_{=1} \wedge \chi\right) \sqcup & \exists u \exists w_{1}^{\circ} \ldots \exists w_{n}^{\circ} \exists w_{1}^{\bullet} \ldots \exists w_{n}^{\bullet} \\
& \forall \vec{z} \exists \vec{w}_{1} \ldots \exists \vec{w}_{n} \exists \vec{w}_{1}^{c} \ldots \exists \vec{w}_{n}^{c}\left(\bigwedge_{i \leq n}\left(\vec{z}=\vec{w}_{i} \vee \vec{z}=\vec{w}_{i}^{c}\right) \wedge \delta^{\prime}\right) .
\end{aligned}
$$

Clearly $\varphi$ is an $\operatorname{EXC}_{L}[k]$-sentence.

Remark. Since we are using the tuples $\vec{w}_{i}$ and $\vec{w}_{i}^{c}$ to simulate a quantified relation and its complement, respectively, it would be natural to add the requirement $\bigwedge_{i \leq n} \vec{w}_{i} \mid \vec{w}_{i}^{c}$ to the sentence $\varphi$ above. However, we will see that this is not necessary, since it suffices that $\vec{w}_{i}$ and $\vec{w}_{i}^{c}$ are quantified in such a way that $X\left(\vec{w}_{i}\right) \cup X\left(\vec{w}_{i}^{c}\right)=M^{k}$ in the resulting team $X$. This condition is achieved by first universally quantifying a tuple $\vec{z}$ and adding disjunction $\vec{z}=\vec{w}_{i} \vee \vec{z}=\vec{w}_{i}^{c}$ for each $i \leq n$ (compare with a similar idea in the sentences of Example 4.1).

We write

$$
V^{*}:=\operatorname{Vr}\left(u w_{1}^{\circ} \ldots w_{n}^{\circ} w_{1}^{\bullet} \ldots w_{n}^{\bullet} \vec{w}_{1} \ldots \vec{w}_{n} \vec{w}_{1}^{c} \ldots \vec{w}_{n}^{c}\right) .
$$

Before proving the claim of this theorem, we prove the following two claims.

Claim 1. Let $\mathcal{M}$ be an L-model with at least two elements. Let $\mu \in \operatorname{Sf}(\delta)$ and let $X$ a team for which $V^{*} \subseteq \operatorname{dom}(X)$ and the following assumptions hold:

$$
\left\{\begin{array}{l}
X\left(\vec{w}_{i}\right) \cup X\left(\vec{w}_{i}^{c}\right)=M^{k} \text { for each } i \leq n . \\
\text { The values of } w_{i}^{\circ}, w_{i}^{\bullet}(i \leq n) \text { and } u \text { are constants in } X .
\end{array}\right.
$$

Let $\mathcal{M}^{\prime}:=\mathcal{M}[\vec{A} / \vec{P}] \quad\left(=\mathcal{M}\left[A_{1} / P_{1}, \ldots, A_{n} / P_{n}\right]\right)$, where

$$
A_{i}= \begin{cases}\emptyset & \text { if } X\left(w_{i}^{\circ}\right)=X(u) \text { and } X\left(w_{i}^{\bullet}\right) \neq X(u) \\ M^{k} & \text { if } X\left(w_{i}^{\bullet}\right)=X(u) \text { and } X\left(w_{i}^{\circ}\right) \neq X(u) \\ X\left(\vec{w}_{i}\right) & \text { else. }\end{cases}
$$

Now the following implication holds:

$$
\text { If } \mathcal{M} \vDash_{X} \mu^{\prime} \text {, then } \mathcal{M}^{\prime} \vDash_{X} \mu \text {. }
$$

We prove this claim by structural induction on $\mu$ :

- If $\mu$ is a literal and $P_{i}$ does not occur in $\mu$ for any $i \leq n$, then the claim holds trivially since $\mu^{\prime}=\mu$.

- Let $\mu=P_{j} \vec{t}$ for some $j \leq n$. Suppose that we have $\mathcal{M} \vDash_{X}\left(P_{j} \vec{t}\right)^{\prime}$, i.e. $\mathcal{M} \vDash_{X}\left(\vec{t} \mid \vec{w}_{j}^{c} \vee w_{j}^{\bullet}=u\right) \wedge w_{j}^{\circ} \neq u$. Because the values of $u, w_{j}^{\circ}$ are constants in $X$ and $\mathcal{M} \vDash_{X} w_{j}^{\circ} \neq u$, we have $X\left(w_{j}^{\circ}\right) \neq X(u)$. If $X\left(w_{j}^{\bullet}\right)=X(u)$, then $A_{j}=M^{k}$ and thus trivially $\mathcal{M}^{\prime} \vDash_{X} P_{j} \vec{t}$. Suppose then that $X\left(w_{j}^{\bullet}\right) \neq X(u)$ whence $A_{j}=X\left(\vec{w}_{j}\right)$. Because the values of $u, w_{j}^{\bullet}$ are constants in $X$ and $\mathcal{M} \vDash_{X} \vec{t} \mid \vec{w}_{j}^{c} \vee w_{j}^{\bullet}=u$, it must be that $\mathcal{M} \vDash_{X} \vec{t} \mid \vec{w}_{j}^{c}$. Now $X(\vec{t}) \cap X\left(\vec{w}_{j}^{c}\right)=\emptyset$ and $X\left(\vec{w}_{j}\right) \cup X\left(\vec{w}_{j}^{c}\right)=M^{k}$. Hence $X(\vec{t}) \subseteq \overline{X\left(\vec{w}_{j}^{c}\right)} \subseteq X\left(\vec{w}_{j}\right)=A_{j}$ and thus $\mathcal{M}^{\prime} \vDash_{X} P_{j} \vec{t}$. 
- Let $\mu=\neg P_{j} \vec{t}$ for some $j \leq n$. Suppose that we have $\mathcal{M} \vDash_{X}\left(\neg P_{j} \vec{t}\right)^{\prime}$, i.e. $\mathcal{M} \vDash_{X}\left(\vec{t} \mid \vec{w}_{j} \vee w_{j}^{\circ}=u\right) \wedge w_{j}^{\bullet} \neq u$. Because the values of $u, w_{j}^{\bullet}$ are constants and $\mathcal{M} \vDash_{X} w_{j}^{\bullet} \neq u$, we have $X\left(w_{j}^{\bullet} \neq X(u)\right.$. If $X\left(w_{j}^{\circ}\right)=X(u)$, then $A_{j}=\emptyset$ and thus trivially $\mathcal{M}^{\prime} \vDash_{X} \neg P_{j} \vec{t}$. Suppose then that $X\left(w_{i}^{\circ}\right) \neq X(u)$ whence $A_{j}=X\left(\vec{w}_{j}\right)$. Because the values of $u, w_{j}^{\circ}$ are constants in $X$ and $\mathcal{M} \vDash_{X} \vec{t} \mid \vec{w}_{j} \vee w_{j}^{\circ}=u$, we have $\mathcal{M} \vDash_{X} \vec{t} \mid \vec{w}_{j}$. Now $X(\vec{t}) \subseteq \overline{X\left(\vec{w}_{j}\right)}=\overline{A_{j}}$ and thus $\mathcal{M}^{\prime} \vDash_{X} \neg P_{j} \vec{t}$.

- The case $\mu=\psi \wedge \theta$ is straightforward to prove.

- Let $\mu=\psi \vee \theta$. Suppose that $\mathcal{M} \vDash_{X}(\psi \vee \theta)^{\prime}$, i.e. $\mathcal{M} \vDash_{X} \psi^{\prime} \underline{\vee}^{\mathbf{U}} \theta^{\prime}$. By Proposition 3.5 there exist $Y_{1}, Y_{2} \subseteq X$ s.t. $Y_{1} \cup Y_{2}=X, \mathcal{M} \vDash_{Y_{1}^{*}} \psi^{\prime}$ and $\mathcal{M} \vDash_{Y_{2}^{*}} \theta^{\prime}$, where

$$
\left\{\begin{array}{l}
Y_{1}^{*}:=Y_{1}\left[X\left(\vec{w}_{1}\right) / \vec{w}_{1}, \ldots, X\left(\vec{w}_{n}\right) / \vec{w}_{n}, X\left(\vec{w}_{1}^{c}\right) / \vec{w}_{1}^{c}, \ldots, X\left(\vec{w}_{n}^{c}\right) / \vec{w}_{n}^{c}\right] \\
Y_{2}^{*}:=Y_{2}\left[X\left(\vec{w}_{1}\right) / \vec{w}_{1}, \ldots, X\left(\vec{w}_{n}\right) / \vec{w}_{n}, X\left(\vec{w}_{1}^{c}\right) / \vec{w}_{1}^{c}, \ldots, X\left(\vec{w}_{n}^{c}\right) / \vec{w}_{n}^{c}\right]
\end{array}\right.
$$

Now the sets of values for $\vec{w}_{i}$ and $\vec{w}_{i}^{c}$ are the same in $Y_{1}^{*}$ and $Y_{2}^{*}$ as in $X$. Because the values of $u$ and $w_{i}^{\circ}, w_{i}^{\bullet}$ are constants in $X$ they have (the same) constant values in $Y_{1}^{*}$ and $Y_{2}^{*}$. Hence, by the inductive hypothesis, we have $\mathcal{M}^{\prime} \vDash_{Y_{1}^{*}} \psi$ and $\mathcal{M}^{\prime} \vDash_{Y_{2}^{*}} \theta$. Since none of the variables in $V^{*}$ occurs in $\psi \vee \theta$, by locality $\mathcal{M}^{\prime} \vDash_{Y_{1}} \psi$ and $\mathcal{M}^{\prime} \vDash_{Y_{2}} \theta$. Therefore $\mathcal{M}^{\prime} \vDash_{X} \psi \vee \theta$.

- The cases $\mu=\exists x \psi$ and $\mu=\forall x \psi$ are straightforward to prove. (Note here that, since $x \notin V^{*}$, the assumptions of Claim 1 hold in the resulting team also after the quantification of $x$.)

Claim 2. Let $\mathcal{M}$ be an L-model with at least two elements. Let $\mu \in \operatorname{Sf}(\delta)$ and $X$ be a team such that $\operatorname{dom}(X)=\operatorname{Fr}(\mu)$. Assume that $A_{1}, \ldots, A_{n} \subseteq M^{k}$, $\mathcal{M}^{\prime}:=\mathcal{M}[\vec{A} / \vec{P}]$ and $a, b \in M$ s.t. $a \neq b$. Let

$$
\begin{gathered}
X^{\prime}:=X\left[\{a\} / u, B_{1}^{\circ} / w_{1}^{\circ}, \ldots, B_{n}^{\circ} / w_{n}^{\circ}, B_{1}^{\bullet} / w_{1}^{\bullet}, \ldots, B_{n}^{\bullet} / w_{n}^{\bullet},\right. \\
\text { where } \begin{cases}\left.B_{1} / \vec{w}_{1}, \ldots, B_{n} / \vec{w}_{n}, B_{1}^{c} / \vec{w}_{1}^{c}, \ldots, B_{n}^{c} / \vec{w}_{n}^{c}\right], \\
B_{i}^{\circ}=\{b\}, B_{i}^{\bullet}=\{b\} \text { and } B_{i}=B_{i}^{c}=B^{\circ}=\{a\} \text { and } B_{i}=B_{i}^{c}=M^{k} & \text { if } A_{i}=\emptyset \\
B_{i}^{\circ}=\{b\}, B_{i}^{\bullet}=\{b\}, B_{i}=A_{i} \text { and } B_{i}^{c}=\overline{A_{i}} & \text { else. }\end{cases}
\end{gathered}
$$

Now the following implication holds:

$$
\text { If } \mathcal{M}^{\prime} \vDash_{X} \mu \text {, then } \mathcal{M} \vDash_{X^{\prime}} \mu^{\prime} \text {. }
$$

We prove this claim by structural induction on $\mu$. Note that if $X=\emptyset$, then also $X^{\prime}=\emptyset$ and thus the claim holds by the empty team property. Hence we may assume that $X \neq \emptyset$.

- If $\mu$ is a literal and $P_{i}$ does not occur in $\mu$ for any $i \leq n$, then the claim holds by locality since $\mu^{\prime}=\mu$. 
- Let $\mu=P_{j} \vec{t}$ for some $j \leq n$. Suppose $\mathcal{M}^{\prime} \vDash_{X} P_{j} \vec{t}$, i.e. $X(\vec{t}) \subseteq P_{j}^{\mathcal{M}^{\prime}}=A_{j}$. Since $X \neq \emptyset$, also $X(\vec{t}) \neq \emptyset$ and thus $A_{j} \neq \emptyset$. Hence $X^{\prime}\left(w_{j}^{\circ}\right)=\{b\}$, and thus $\mathcal{M} \vDash_{X^{\prime}} w_{i}^{\circ} \neq u$ since $X^{\prime}(u)=\{a\}$. If $A_{j}=M^{k}$, then $X^{\prime}\left(w_{i}^{\bullet}\right)=\{a\}$ and thus $\mathcal{M} \vDash_{X^{\prime}} w_{j}^{\bullet}=u$, whence $\mathcal{M} \vDash_{X^{\prime}}\left(\vec{t} \mid \vec{w}_{j}^{c} \vee w_{j}^{\bullet}=u\right) \wedge w_{j}^{\circ} \neq u$, i.e. $\mathcal{M} \vDash_{X^{\prime}}\left(P_{j} \vec{t}\right)^{\prime}$. Suppose then that $A_{j} \neq M^{k}$. Now we have $X^{\prime}\left(\vec{w}_{j}^{c}\right)=\overline{A_{j}}$, i.e. $\overline{X^{\prime}\left(w_{j}^{c}\right)}=A_{j}$, and thus $X^{\prime}(\vec{t})=X(\vec{t}) \subseteq A_{j}=\overline{X^{\prime}\left(\vec{w}_{j}^{c}\right)}$. Hence we have $\mathcal{M} \vDash_{X^{\prime}} \vec{t} \mid \vec{w}_{j}^{c}$ and thus $\mathcal{M} \vDash_{X^{\prime}}\left(\vec{t} \mid \vec{w}_{j}^{c} \vee w_{j}^{\bullet}=u\right) \wedge w_{j}^{\circ} \neq u$, i.e. $\mathcal{M} \vDash_{X^{\prime}}\left(P_{j} \vec{t}\right)^{\prime}$.

- Let $\mu=\neg P_{j} \vec{t}$ for some $j \leq n$. Suppose that we have $\mathcal{M}^{\prime} \vDash_{X} \neg P_{j} \vec{t}$, i.e. $X(\vec{t}) \subseteq \overline{P_{j}^{\mathcal{M}^{\prime}}}=\overline{A_{j}}$. Since $X \neq \emptyset$, we have $X(\vec{t}) \neq \emptyset$ and thus $\overline{A_{j}} \neq \emptyset$, i.e. $A_{j} \neq M^{k}$. Hence $X^{\prime}\left(w_{j}^{\bullet}\right)=\{b\}$, and thus $\mathcal{M} \vDash_{X^{\prime}} w_{i}^{\bullet} \neq u$ since $X^{\prime}(u)=\{a\}$. If $A_{j}=\emptyset$, then $X^{\prime}\left(w_{i}^{\circ}\right)=\{a\}$ and thus $\mathcal{M} \vDash_{X^{\prime}} w_{j}^{\circ}=u$, whence $\mathcal{M} \vDash_{X^{\prime}}\left(\vec{t} \mid \vec{w}_{j} \vee w_{j}^{\circ}=u\right) \wedge w_{j}^{\bullet} \neq u$, i.e. $\mathcal{M} \vDash_{X^{\prime}}\left(\neg P_{j} \vec{t}\right)^{\prime}$. Suppose then that we have $A_{j} \neq \emptyset$. Then $X^{\prime}\left(\vec{w}_{j}\right)=A_{j}$ and thus it holds that $X^{\prime}(\vec{t})=X(\vec{t}) \subseteq \overline{A_{j}}=\overline{X^{\prime}\left(\vec{w}_{j}\right)}$. Hence we have $\mathcal{M} \vDash_{X^{\prime}} \vec{t} \mid \vec{w}_{j}$ and therefore $\mathcal{M} \vDash_{X^{\prime}}\left(\vec{t} \mid \vec{w}_{j} \vee w_{j}^{\circ}=u\right) \wedge w_{j}^{\bullet} \neq u$, i.e. $\mathcal{M} \vDash_{X^{\prime}}\left(\neg P_{j} \vec{t}\right)^{\prime}$.

- The case $\mu=\psi \wedge \theta$ is straightforward to prove.

- Let $\mu=\psi \vee \theta$. Suppose that $\mathcal{M}^{\prime} \vDash_{X} \psi \vee \theta$, i.e. there exist $Y_{1}, Y_{2} \subseteq X$ s.t. $Y_{1} \cup Y_{2}=X, \mathcal{M}^{\prime} \vDash_{Y_{1}} \psi$ and $\mathcal{M}^{\prime} \vDash_{Y_{2}} \theta$. Let $Y_{1}^{\prime}, Y_{2}^{\prime}$ be the teams obtained by extending the teams $Y_{1}, Y_{2}$ as $X^{\prime}$ is obtained by extending $X$. Then, by the inductive hypothesis, we have $\mathcal{M} \vDash_{Y_{1}^{\prime}} \psi^{\prime}$ and $\mathcal{M} \vDash_{Y_{2}^{\prime}} \theta^{\prime}$. Now the following holds:

$$
\left\{\begin{array}{l}
Y_{1}^{\prime}=Y_{1}^{\prime}\left[X^{\prime}\left(\vec{w}_{1}\right) / \vec{w}_{1}, \ldots, X^{\prime}\left(\vec{w}_{n}\right) / \vec{w}_{n}, X^{\prime}\left(\vec{w}_{1}^{c}\right) / \vec{w}_{1}^{c}, \ldots, X^{\prime}\left(\vec{w}_{n}^{c}\right) / \vec{w}_{n}^{c}\right] \\
Y_{2}^{\prime}=Y_{2}^{\prime}\left[X^{\prime}\left(\vec{w}_{1}\right) / \vec{w}_{1}, \ldots, X^{\prime}\left(\vec{w}_{n}\right) / \vec{w}_{n}, X^{\prime}\left(\vec{w}_{1}^{c}\right) / \vec{w}_{1}^{c}, \ldots, X^{\prime}\left(\vec{w}_{n}^{c}\right) / \vec{w}_{n}^{c}\right] .
\end{array}\right.
$$

Note that also $Y_{1}^{\prime}, Y_{2}^{\prime} \subseteq X^{\prime}$ and $Y_{1}^{\prime} \cup Y_{2}^{\prime}=X^{\prime}$. Thus by Proposition 3.5 $\mathcal{M} \vDash_{X^{\prime}} \psi^{\prime} \underline{\vee}^{\mathbf{U}} \theta^{\prime}$, i.e. $\mathcal{M} \vDash_{X^{\prime}}(\psi \vee \theta)^{\prime}$.

- Let $\mu=\exists x \psi$ (the case $\mu=\forall x \psi$ is proven similarly). Suppose that $\mathcal{M}^{\prime} \vDash_{X} \exists x \psi$, i.e. there exists $F: X \rightarrow M$ s.t. $\mathcal{M}^{\prime} \vDash_{X[F / x]} \psi$. Let $F^{\prime}$ : $X^{\prime} \rightarrow M$ such that $s \mapsto F(s \uparrow \operatorname{Fr}(\mu))$ for each $s \in X^{\prime}$. Note that $F^{\prime}$ is well defined since $\operatorname{dom}(X)=\operatorname{Fr}(\mu)$ by the assumption.

Let $(X[F / x])^{\prime}$ be a team that is obtained by extending the team $X[F / x]$ analogously as $X^{\prime}$ is obtained by extending $X$. Now by inductive hypothesis we have $\mathcal{M} \vDash_{(X[F / x])^{\prime}} \psi^{\prime}$. By the definition of $F^{\prime}$ it is easy to see that $(X[F / x])^{\prime}=X^{\prime}\left[F^{\prime} / x\right]$ and thus $\mathcal{M} \vDash_{X^{\prime}\left[F^{\prime} / x\right]} \psi^{\prime}$. Hence we have $\mathcal{M} \vDash_{X^{\prime}} \exists x \psi^{\prime}$, i.e. $\mathcal{M} \vDash_{X^{\prime}}(\exists x \psi)^{\prime}$.

We are now ready to prove the claim of this theorem:

$$
\mathcal{M} \vDash \varphi \text { iff } \mathcal{M} \vDash \Phi .
$$

Suppose first that $\mathcal{M} \vDash \varphi$, i.e. $\mathcal{M} \vDash \gamma_{=1} \wedge \chi$ or

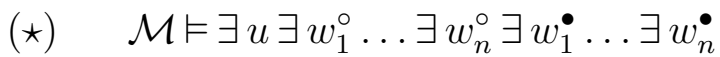

$$
\forall \vec{z} \exists \vec{w}_{1} \ldots \exists \vec{w}_{n} \exists \vec{w}_{1}^{c} \ldots \exists \vec{w}_{n}^{c}\left(\bigwedge_{i \leq n}\left(\vec{z}=\vec{w}_{i} \vee \vec{z}=\vec{w}_{i}^{c}\right) \wedge \delta^{\prime}\right)
$$


If $\mathcal{M} \vDash \gamma_{=1} \wedge \chi$, the claim holds by Lemma 4.4. Suppose then $(\star)$, whence by the (strict) semantics of existential quantifier there are $a, b_{1} \ldots b_{n}, b_{1}^{\prime}, \ldots, b_{n}^{\prime} \in M$ such that

$$
\mathcal{M} \vDash_{X_{1}} \forall \vec{z} \exists \vec{w}_{1} \ldots \exists \vec{w}_{n} \exists \vec{w}_{1}^{c} \ldots \exists \vec{w}_{n}^{c}\left(\bigwedge_{i \leq n}\left(\vec{z}=\vec{w}_{i} \vee \vec{z}=\vec{w}_{i}^{c}\right) \wedge \delta^{\prime}\right),
$$

where $X_{1}:=\left\{\emptyset\left[a / u, b_{1} / w_{1}^{\circ}, \ldots, b_{n} / w_{n}^{\circ}, b_{1}^{\prime} / w_{1}^{\bullet}, \ldots, b_{n}^{\prime} / w_{n}^{\bullet}\right]\right\}$. Note that since $X_{1}$ consists only of a single assignment, the values of $u, w_{i}^{\circ}$ and $w_{i}^{\bullet}(i \leq n)$ are trivially constants in the team $X_{1}$. Let $X_{2}:=X_{1}\left[M^{k} / \vec{z}\right]$. Now there exist functions $\mathcal{F}_{i}: X_{2}\left[\mathcal{F}_{1} / \vec{w}_{1}, \ldots, \mathcal{F}_{i-1} / \vec{w}_{i-1}\right] \rightarrow M^{k}$ such that

$$
\mathcal{M} \vDash_{X_{3}} \exists \vec{w}_{1}^{c} \ldots \exists \vec{w}_{n}^{c}\left(\bigwedge_{i \leq n}\left(\vec{z}=\vec{w}_{i} \vee \vec{z}=\vec{w}_{i}^{c}\right) \wedge \delta^{\prime}\right),
$$

where $X_{3}:=X_{2}\left[\mathcal{F}_{1} / \vec{w}_{1}, \ldots, \mathcal{F}_{n} / \vec{w}_{n}\right]$.

Furthermore there exist functions $\mathcal{F}_{i}^{\prime}: X_{3}\left[\mathcal{F}_{1}^{\prime} / \vec{w}_{1}^{c}, \ldots, \mathcal{F}_{i-1}^{\prime} / \vec{w}_{i-1}^{c}\right] \rightarrow M^{k}$ such that $\mathcal{M} \vDash_{X_{4}} \wedge_{i \leq n}\left(\vec{z}=\vec{w}_{i} \vee \vec{z}=\vec{w}_{i}^{c}\right) \wedge \delta^{\prime}$, where $X_{4}:=X_{3}\left[\mathcal{F}_{1}^{\prime} / \vec{w}_{1}^{c}, \ldots, \mathcal{F}_{n}^{\prime} / \vec{w}_{n}^{c}\right]$. Since $X_{4}(\vec{z})=M^{k}$ and $\mathcal{M} \vDash_{X_{4}} \bigwedge_{i \leq n}\left(\vec{z}=\vec{w}_{i} \vee \vec{z}=\vec{w}_{i}^{c}\right)$, it is easy to see that $X_{4}\left(\vec{w}_{i}\right) \cup X_{4}\left(\vec{w}_{i}^{c}\right)=M^{k}$ for each $i \leq n$. Now all the assumptions of Claim 1 hold for the team $X_{4}$. Let $\mathcal{M}^{\prime}:=\mathcal{M}[\vec{A} / \vec{P}]$, where

$$
A_{i}= \begin{cases}\emptyset & \text { if } X_{4}\left(w_{i}^{\circ}\right)=X_{4}(u) \text { and } X_{4}\left(w_{i}^{\bullet}\right) \neq X_{4}(u) \\ M^{k} & \text { if } X_{4}\left(w_{i}^{\bullet}\right)=X_{4}(u) \text { and } X_{4}\left(w_{i}^{\circ}\right) \neq X_{4}(u) \\ X_{4}\left(\vec{w}_{i}\right) & \text { else. }\end{cases}
$$

Since $\mathcal{M} \vDash_{X_{4}} \delta^{\prime}$, by Claim 1 we have $\mathcal{M}^{\prime} \vDash_{X_{4}} \delta$. By locality $\mathcal{M}^{\prime} \vDash \delta$, and therefore $\mathcal{M} \vDash \Phi$.

Suppose then that $\mathcal{M} \vDash \Phi$. If $|M|=1$, then by Lemma 4.4 we have $\mathcal{M} \vDash \gamma_{=1} \wedge \chi$ and thus $\mathcal{M} \vDash \varphi$. Hence we may assume that $|M| \geq 2$, whence there exist $a, b \in M$ s.t. $a \neq b$. Since $\mathcal{M} \vDash \Phi$, there exist $A_{1}, \ldots, A_{n} \subseteq M^{k}$ s.t. $\mathcal{M}[\vec{A} / \vec{P}] \vDash \delta$. Let

$$
\begin{array}{r}
X^{\prime}:=\{\emptyset\}\left[\{a\} / u, B_{1}^{\circ} / w_{1}^{\circ}, \ldots, B_{n}^{\circ} / w_{n}^{\circ}, B_{1}^{\bullet} / w_{1}^{\bullet}, \ldots, B_{n}^{\bullet} / w_{n}^{\bullet},\right. \\
\left.B_{1} / \vec{w}_{1}, \ldots, B_{n} / \vec{w}_{n}, B_{1}^{c} / \vec{w}_{1}^{c}, \ldots, B_{n}^{c} / \vec{w}_{n}^{c}\right],
\end{array}
$$

where $B_{i}^{\circ}, B_{i}^{\bullet}, B_{i}, B_{i}^{c}(i \leq n)$ are defined as in the assumptions of Claim 2, Since $\mathcal{M}[\vec{A} / \vec{P}] \vDash \delta$, by Claim 2 we have $\mathcal{M} \vDash_{X^{\prime}} \delta^{\prime}$. Let

$$
\begin{aligned}
\mathcal{F}:\{\emptyset\} \rightarrow M^{2 n+1}, \quad \emptyset \mapsto a b_{1} \ldots b_{n} b_{1}^{\prime} \ldots b_{n}^{\prime}, & \text { where }\left\{\begin{array} { l } 
{ b _ { i } = a \text { if } A _ { i } = \emptyset } \\
{ b _ { i } = b \text { else } }
\end{array} \text { and } \left\{\begin{array}{l}
b_{i}^{\prime}=a \text { if } A_{i}=M^{k} \\
b_{i}^{\prime}=b \text { else. }
\end{array}\right.\right.
\end{aligned}
$$

Let $X_{1}:=\{\emptyset\}\left[\mathcal{F} / u w_{1}^{\circ} \ldots w_{n}^{\circ} w_{1}^{\bullet} \ldots w_{n}^{\bullet}\right]$ and let $X_{2}:=X_{1}\left[M^{k} / \vec{z}\right]$. We fix some $\vec{b}_{i} \in A_{i}$ for each $i \leq n$ for which $A_{i} \neq \emptyset$ and define the functions

$$
\mathcal{F}_{i}: X_{2}\left[\mathcal{F}_{1} / \vec{w}_{1}, \ldots, \mathcal{F}_{i-1} / \vec{w}_{i-1}\right] \rightarrow M^{k}, \quad \begin{cases}s \mapsto s(\vec{z}) & \text { if } s(\vec{z}) \in A_{i} \text { or } A_{i}=\emptyset \\ s \mapsto \vec{b}_{i} & \text { else. }\end{cases}
$$


Let $X_{3}:=X_{2}\left[\mathcal{F}_{1} / \vec{w}_{1}, \ldots, \mathcal{F}_{n} / \vec{w}_{n}\right]$. We fix some $\vec{b}_{i}^{\prime} \in \overline{A_{i}}$ for each $i \leq n$ for which $A_{i} \neq M^{k}$ and define

$$
\mathcal{F}_{i}^{\prime}: X_{3}\left[\mathcal{F}_{1}^{\prime} / \vec{w}_{1}^{c}, \ldots, \mathcal{F}_{i-1}^{\prime} / \vec{w}_{i-1}^{c}\right] \rightarrow M^{k}, \begin{cases}s \mapsto s(\vec{z}) & \text { if } s(\vec{z}) \in \overline{A_{i}} \text { or } A_{i}=M^{k} \\ s \mapsto \overrightarrow{b_{i}^{\prime}} & \text { else. }\end{cases}
$$

Let $X_{4}:=X_{3}\left[\mathcal{F}_{1}^{\prime} / \vec{w}_{1}^{c}, \ldots, \mathcal{F}_{n}^{\prime} / \vec{w}_{n}^{c}\right]$. By the definitions of the functions $\mathcal{F}_{i}, \mathcal{F}_{i}^{\prime}$ it is quite easy to see that $\mathcal{M} \vDash_{X_{4}} \bigwedge_{i \leq n}\left(\vec{z}=\vec{w}_{i} \vee \vec{z}=\vec{w}_{i}^{c}\right)$. By the definitions of the choice functions for the variables in $V^{*}$, we observe that $X_{4} \uparrow V^{*} \subseteq X^{\prime}$ (note here that the variables in $\vec{z}$ are not in $\operatorname{dom}\left(X^{\prime}\right)$ ). Hence by locality and downwards closure $\mathcal{M} \vDash_{X_{4}} \delta^{\prime}$. Thus $\mathcal{M} \vDash_{X_{4}} \bigwedge_{i \leq n}\left(\vec{z}=\vec{w}_{i} \vee \vec{z}=\vec{w}_{i}^{c}\right) \wedge \delta^{\prime}$ and furthermore $\mathcal{M} \vDash \varphi$.

Corollary 4.6. On the level of sentences $\mathrm{EXC}[k] \equiv \operatorname{ESO}[k]$.

Proof. In [19] we have presented a translation from $\mathrm{EXC}[k]$ to $\mathrm{ESO}[k]$. By Theorem 4.5, on the level of sentences, there is also a translation from $\operatorname{ESO}[k]$ to $\mathrm{EXC}[k]$.

In particular, we can capture existential monadic second order logic, EMSO, by using unary exclusion atoms. This is particularly interesting since EMSO cannot be captured with any arity fragment of dependence nor independence logic (as a consequence by results in [3, 6]). Hence we argue that exclusion logic deserves extra recognition by capturing this important fragment of ESO.

\subsection{Relationship between $\mathrm{INC}[k]$ and $\mathrm{EXC}[k]$}

Since by [19] INEX $[k]$ captures ESO $[k]$, by Corollary 4.6 we can deduce that $\mathrm{INEX}[k] \equiv \mathrm{EXC}[k]$ on the level of sentences. Hence, on the level of sentences, $k$-ary inclusion atoms do not increase the expressive power of $\operatorname{EXC}[k]$.

By Dawar [2], 3-colorability of a graph cannot be expressed in fixed point logic. Since by [7] INC is equivalent with positive greatest fixed point logic, this property is not expressible in INC. However, since it can be expressed in $\mathrm{EXC}[1]$ (recall Example 4.1), INC $[k]$ is strictly weaker than $\mathrm{EXC}[k]$ on the level of sentences for any $k$.

Corollary 4.7. On the level of sentences $\operatorname{INC}[k]<\operatorname{EXC}[k]$ for any $k \geq 1$.

This consequence is somewhat surprising since inclusion and exclusion atoms can be seen as duals of each other ([19]). As a matter of fact, exclusion atoms can also be simulated with inclusion atoms in an analogous way as we simulated inclusion atoms with exclusion atoms. To see this, suppose that $X$ is a team and $\vec{x}, \vec{w}, \vec{w}^{c}$ are tuples variables s.t. $X\left(\vec{w}^{c}\right)=\overrightarrow{X(\vec{w})}$. Now we have: $\mathcal{M} \vDash_{X} \vec{x} \mid \vec{w}$ iff $\mathcal{M} \vDash_{X} \vec{x} \subseteq \vec{w}^{c}$ (c.f. the observation in the beginning of Section 4.2).

By the observation above, it would be natural to assume that $\operatorname{ESO}_{L}[k]$ sentences could be expressed with INC $[k]$-sentences similarly as we did with $\mathrm{EXC}[k]$-sentences. But this is impossible as we deduced above. The problem is that in INC there is no way to "force" the tuples $\vec{w}$ and $\vec{w}^{c}$ to be quantified in such a way that their values would be complements of each other. However, there is a possibility this could be done in inclusion logic with strict semantics, since Galliani, Hannula and Kontinen [6] have shown that this logic is equivalent with ESO. We will study this question in the next section. 


\section{Lower bound for the expressive power of $k$-ary inclusion logic with strict semantics}

In this section we will study the expressive power of $k$-ary inclusion logic with strict semantics, denoted by $\mathrm{INC}^{s}[k]$. By using similar tricks as in the previous section, we can formulate a translation from $\mathrm{ESO}[k]$ to $\mathrm{INC}^{s}[k]$ and thus obtain a lower bound for the expressive power of $\operatorname{INC}^{s}[k]$.

In this section we will exclusively use strict-semantics - both for evaluating existential quantifiers and for evaluating disjunctions. In order to make make this more explicit, we could have chosen to use a different symbol for the truth - such as $\vDash^{s}$. But have we decided keep our notation more simple.

\subsection{Properties of inclusion logic with strict semantics}

Inclusion logic with the alternative (nonequivalent) strict semantics has been studied in e.g. [6] and [10]. As we have noted before, when using strict semantics with inclusion logic, we lose the locality property. Hence the resulting logic is a bit strange by having some counterintuitive properties 4 . We have to be extra careful when formulating our proofs for $\mathrm{INC}^{s}$ since locality is one of the most commonly used properties used in proofs in the framework of team semantics.

Moreover, not only locality of INC is lost with strict semantics. With inclusion logic we very often use its property of being closed under unions. But also this property is lost with strict semantics, as seen by the following example $5^{5}$

Example 5.1. The first case below shows that, with strict semantics for disjunction, the closure under unions is lost for INC. The second case shows the same for strict semantics for existential quantifier. For both cases, let $M=\{0,1,2\}$.

1. Let $\varphi:=x \subseteq y \vee y \subseteq x$ and let $X_{1}=\left\{s_{0}, s_{1}\right\}$ and $X_{2}=\left\{s_{0}, s_{2}\right\}$, where

$$
\left\{\begin{array} { l } 
{ s _ { 0 } ( x ) = 0 } \\
{ s _ { 0 } ( y ) = 0 }
\end{array} \quad \left\{\begin{array} { l } 
{ s _ { 1 } ( x ) = 0 } \\
{ s _ { 1 } ( y ) = 1 }
\end{array} \quad \left\{\begin{array}{l}
s_{2}(x)=2 \\
s_{2}(y)=0 .
\end{array}\right.\right.\right.
$$

Now $\mathcal{M} \vDash_{X_{1}} \varphi$ since we do a trivial splitting of $X_{1}$ by leaving the right side empty. Similarly $\mathcal{M} \vDash_{X_{2}} \varphi$ since we can leave the left side empty when splitting $X_{2}$. But $\mathcal{M} \not \nvdash_{X_{1} \cup X_{2}} \varphi$ since there is no way to split $X_{1} \cup X_{2}=$ $\left\{s_{0}, s_{1}, s_{2}\right\}$ into two disjoint subteams such that the other would satisfy $x \subseteq y$ and the other would satisfy $y \subseteq x$. (Note that with lax semantics $X_{1} \cup X_{2}$ can here be split into the subteams $\left\{s_{0}, s_{1}\right\}$ and $\left\{s_{0}, s_{2}\right\}$.)

2. Let $\varphi:=\exists z(z \neq x \wedge z \neq y \wedge x \subseteq z)$ and let $X_{1}=\left\{s_{0}, s_{1}\right\}$ and $X_{2}=$ $\left\{s_{0}, s_{2}\right\}$, where

$$
\left\{\begin{array} { l } 
{ s _ { 0 } ( x ) = 0 } \\
{ s _ { 0 } ( y ) = 0 }
\end{array} \quad \left\{\begin{array} { l } 
{ s _ { 1 } ( x ) = 1 } \\
{ s _ { 1 } ( y ) = 2 }
\end{array} \quad \left\{\begin{array}{l}
s_{2}(x)=2 \\
s_{2}(y)=1
\end{array}\right.\right.\right.
$$

\footnotetext{
${ }^{4}$ Note that IF-logic is not local either. This is manifested by some exotic properties, such as signaling.

${ }^{5}$ The corresponding observation has been done independently in [1] for propositional inclusion logic.
} 
Now $\mathcal{M} \vDash_{X_{1}} \varphi$ since we can map $s_{0}$ to 1 and $s_{1}$ to 0 . Similarly $\mathcal{M} \vDash_{X_{2}} \varphi$ since we can map $s_{0}$ to 2 and $s_{2}$ to 0 . However, $\mathcal{M} \nvdash_{X_{1} \cup X_{2}} \varphi$ since $\left|\left(X_{1} \cup X_{2}\right)(x)\right|=3$, but both $s_{1}$ and $s_{2}$ must be mapped to 0 . (Note that with lax semantics $s_{0}$ can here be mapped to both 1 and 2.)

\subsection{Simulating exclusion in $\mathrm{INC}^{s}$}

In order to formulate a translation from $\operatorname{ESO}[k]$ to $\operatorname{INC}^{s}[k]$, we need to be

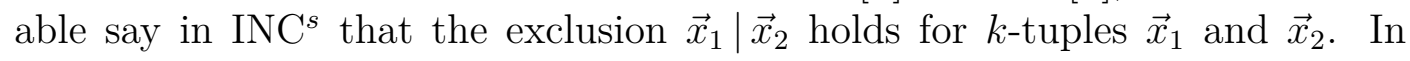
certain cases this is possible; even without access to the complementary values of $\vec{x}_{1}$ and $\vec{x}_{2}$ in the team. For this purpose, we consider a variant of termvalue preserving disjunction ([19]). The disjunction $\varphi_{\vec{x}_{1}} \vee_{\vec{x}_{2}} \psi$ states the same as normal disjunction, with the additional assumption that the values of $\vec{x}_{1}$ are preserved on the left and the values of $\vec{x}_{2}$ on the right when the team is split. That is, $\mathcal{M} \vDash_{X} \varphi_{\vec{x}_{1}} \vee_{\vec{x}_{2}} \psi$ holds if and only if there are $Y, Y^{\prime} \subseteq X$ such that $Y \cup Y^{\prime}=X, Y \cap Y^{\prime}=\emptyset, \mathcal{M} \vDash_{Y} \varphi, \mathcal{M} \vDash_{Y^{\prime}} \psi$ and additionally $Y\left(\vec{x}_{1}\right)=X\left(\vec{x}_{1}\right)$ and $Y^{\prime}\left(\vec{x}_{2}\right)=X\left(\vec{x}_{2}\right)$.

When $\varphi:=\vec{x}_{1} \subseteq \vec{z}$ and $\psi:=\vec{x}_{2} \subseteq \vec{z}$, the truth of $\varphi_{\vec{x}_{1}} \vee_{\vec{x}_{2}} \psi$ (by strict semantics) will guarantee in certain teams that the exclusion $\vec{x}_{1} \mid \vec{x}_{2}$ holds. Sufficient condition here is that all the values of all variables in $X$ are dependent on the values of $\vec{z}$. When this holds and $X$ is split into disjoint subteams $Y$ and $Y^{\prime}$, it is then guaranteed that $Y(\vec{z}) \cap Y^{\prime}(\vec{z})=\emptyset$. Supposing that $\mathcal{M} \vDash_{X} \vec{x}_{1} \subseteq \vec{z}_{\vec{x}_{1}} \vee_{\vec{x}_{2}} \vec{x}_{2} \subseteq \vec{z}$, we then have $X\left(\vec{x}_{1}\right)=Y\left(\vec{x}_{1}\right) \subseteq Y(\vec{z})$ and $X\left(\vec{x}_{1}\right)=Y^{\prime}\left(\vec{x}_{1}\right) \subseteq Y^{\prime}(\vec{z})$, whence it follows that $X\left(\vec{x}_{1}\right) \cap X\left(\vec{x}_{2}\right)=\emptyset$.

In Definition 5.1 the defined operator $\operatorname{exc}_{c_{l}, c_{r}, \vec{z}}\left(\vec{x}_{1}, \vec{x}_{2}\right)$ is derived quite directly from the the definition of the disjunction $\vec{x}_{1} \subseteq \vec{z}_{\vec{x}_{1}} \vee_{\vec{x}_{2}} \vec{x}_{2} \subseteq \vec{z}$ in INEX. The definition is very complex, but we try to explain its main idea here briefly. Suppose that $c_{l}, c_{r}$ have constant values in a team $X$ and that $X\left(c_{l}\right) \neq X\left(c_{r}\right) \cdot{ }^{6}$ Now we can quantify a "label variable" $y$ for each assignment such that it gets either the value of $c_{l}$ or $c_{r}$. This value states whether the assignment in question will be placed on the left $\left(c_{l}\right)$ or on the right $\left(c_{r}\right)$ when evaluating a disjunction that follows this quantification. Since these label values are given before the team is split, we can "check" beforehand by using inclusion atoms that the values of tuple $\vec{x}_{1}$ are preserved on the left and the values of $\vec{x}_{2}$ are preserved on the right. This is done with formulas $\theta$ and $\theta^{\prime}$ : the truth of $\theta$ guarantees the preservation of all values except for a constant $\vec{c}_{l}$ and the truth of $\theta^{\prime}$ guarantees the preservation for all values except for a constant $\vec{c}_{r}$. When $\vec{c}_{l} \neq \vec{c}_{r}$, the truth of the conjunction $\theta \wedge \theta^{\prime}$ guarantees the preservation of all values.

Definition 5.1. Let $c_{l}$ and $c_{r}$ be variables and let $\vec{z}, \vec{x}_{1}, \vec{x}_{2}$ be $k$-tuples of

\footnotetext{
${ }^{6}$ With unary dependence atoms $=(x)$ we could state the values for these variables in the team are constants. However, since we cannot express these atoms with inclusion atoms, we have to assume this to be the case. (Alternatively we could use some constant symbols which have different interpretations.)
} 
variables. We write

$$
\begin{gathered}
\operatorname{exc}_{c_{l}, c_{r}, \vec{z}}\left(\vec{x}_{1}, \vec{x}_{2}\right):=\exists y\left(\left(\left(y=c_{l} \wedge \vec{x}_{1} \subseteq \vec{z}\right) \vee\left(y=c_{r} \wedge \vec{x}_{2} \subseteq \vec{z}\right)\right) \wedge \theta \wedge \theta^{\prime}\right), \\
\theta:=\exists \vec{z}_{1} \exists \vec{z}_{2}\left(\left(\left(y=c_{l} \wedge \vec{z}_{1}=\vec{x}_{1} \wedge \vec{z}_{2}=\vec{c}_{1}\right)\right.\right. \\
\left.\left.\vee\left(y=c_{r} \wedge \vec{z}_{1}=\vec{c}_{1} \wedge \vec{z}_{2}=\vec{x}_{2}\right)\right) \wedge \vec{x}_{1} \subseteq \vec{z}_{1} \wedge \vec{x}_{2} \subseteq \vec{z}_{2}\right) \\
\theta^{\prime}:=\exists \vec{z}_{1} \exists \vec{z}_{2}\left(\left(\left(y=c_{l} \wedge \vec{z}_{1}=\vec{x}_{1} \wedge \vec{z}_{2}=\vec{c}_{2}\right)\right.\right. \\
\left.\left.\vee\left(y=c_{r} \wedge \vec{z}_{1}=\vec{c}_{2} \wedge \vec{z}_{2}=\vec{x}_{2}\right)\right) \wedge \vec{x}_{1} \subseteq \vec{z}_{1} \wedge \vec{x}_{2} \subseteq \vec{z}_{2}\right)
\end{gathered}
$$

where $y$ is a fresh variable, $\vec{z}_{1}, \vec{z}_{2}$ are $k$-tuples of fresh variables and $\vec{c}_{1}$ and $\vec{c}_{2}$ are $k$-tuples such that $\vec{c}_{1}=c_{l} \ldots c_{l}$ and $\vec{c}_{2}=c_{r} \ldots c_{r}$.

The following lemma gives sufficient conditions for the truth of $\mathcal{M} \vDash_{X} \vec{x}_{1} \mid \vec{x}_{2}$. This result is needed when proving Theorem 5.3 in the next section.

Lemma 5.1. Let $\mathcal{M}$ be a model and let $X$ be a team, where $c_{l}$ and $c_{r}$ have different constant values $a$ and $b$, respectively. Suppose that the $k$-tuples $\vec{z}, \vec{x}_{1}$, $\vec{x}_{2}$ are all in $\operatorname{dom}(X)$ and that the variable $y$ in the definition of $\operatorname{exc}_{c_{l}, c_{r}, \vec{z}}\left(\vec{x}_{1}, \vec{x}_{2}\right)$ is not in $\operatorname{dom}(X)$. Moreover, assume that the following conditions hold for $X$.

1. $\mathcal{M} \vDash_{X}=(\vec{z}, v)$ for all $v \in \operatorname{dom}(X)$.

2. $\mathcal{M} \vDash_{X} \operatorname{exc}_{c_{l}, c_{r}, \vec{z}}\left(\vec{x}_{1}, \vec{x}_{2}\right)$.

Then it holds that $X\left(\vec{x}_{1}\right) \cap X\left(\vec{x}_{2}\right)=\emptyset$.

Proof. We write $\vec{a}:=a \ldots a$ and $\vec{b}:=b \ldots b$. Since $\mathcal{M} \vDash_{X} \operatorname{exc}_{c_{l}, c_{r}, \vec{z}}\left(\vec{x}_{1}, \vec{x}_{2}\right)$, there is $F: X \rightarrow M$ s.t. $\mathcal{M} \vDash_{X^{\prime}}\left(\left(y=c_{l} \wedge \vec{x}_{1} \subseteq \vec{z}\right) \vee\left(y=c_{r} \wedge \vec{x}_{2} \subseteq \vec{z}\right)\right) \wedge \theta \wedge \theta^{\prime}$, where $X^{\prime}=X[F / y]$. Thus $\mathcal{M} \vDash_{X^{\prime}} \theta, \mathcal{M} \vDash_{X^{\prime}} \theta^{\prime}$ and there are $Y, Y^{\prime} \subseteq X^{\prime}$ s.t. $Y \cup Y^{\prime}=X^{\prime}, Y \cap Y^{\prime}=\emptyset, \mathcal{M} \vDash_{Y} y=c_{l} \wedge \vec{x}_{1} \subseteq \vec{z}$ and $\mathcal{M} \vDash_{Y^{\prime}} y=c_{r} \wedge \vec{x}_{2} \subseteq \vec{z}$. Since $X^{\prime}\left(c_{l}\right)=\{a\}$ and $X^{\prime}\left(c_{r}\right)=\{b\}$, it is easy to see that the following conditions hold for any assignment $s \in X^{\prime}$ :

$$
s \in Y \text { iff } s(y)=a \quad \text { and } \quad s \in Y^{\prime} \text { iff } s(y)=b .
$$

We first show that $Y(\vec{z}) \cap Y^{\prime}(\vec{z})=\emptyset$. Suppose, for the sake of contradiction, that $Y(\vec{z}) \cap Y^{\prime}(\vec{z}) \neq \emptyset$, whence there is $s \in Y$ and $s^{\prime} \in Y^{\prime}$ s.t. $s(\vec{z})=s^{\prime}(\vec{z})$. Now $s(y)=a$ and $s^{\prime}(y)=b$. Since $\mathcal{M} \vDash_{X}=(\vec{z}, v)$ for all $v \in \operatorname{dom}(X)$, by the strict semantics of existential quantifier we must have $\mathcal{M} \vDash_{X^{\prime}}=(\vec{z}, y)$. But this is impossible since $s(\vec{z})=s^{\prime}(\vec{z})$ and $s(y) \neq s^{\prime}(y)$.

Since $\mathcal{M} \vDash_{X^{\prime}} \theta$, there are $\mathcal{F}_{1}: X^{\prime} \rightarrow M^{k}$ and $\mathcal{F}_{2}: X^{\prime}\left[\mathcal{F}_{1} / \vec{z}_{1}\right] \rightarrow M^{k}$ s.t. $\mathcal{M} \vDash_{Z}\left(\left(y=c_{l} \wedge \vec{z}_{1}=\vec{x}_{1} \wedge \vec{z}_{2}=\vec{c}_{1}\right) \vee\left(y=c_{r} \wedge \vec{z}_{1}=\vec{c}_{1} \wedge \vec{z}_{2}=\vec{x}_{2}\right)\right) \wedge \vec{x}_{1} \subseteq \vec{z}_{1} \wedge \vec{x}_{2} \subseteq \vec{z}_{2}$, where $Z:=X\left[\mathcal{F}_{1} / \vec{z}_{1}, \mathcal{F}_{2} / \vec{z}_{2}\right]$. Hence $\mathcal{M} \vDash_{Z} \vec{x}_{1} \subseteq \vec{z}_{1}, \mathcal{M} \vDash_{Z} \vec{x}_{2} \subseteq \vec{z}_{2}$ and there are $W_{1}, W_{2} \subseteq Z$ s.t. $W_{1} \cup W_{2}=Z, W_{1} \cap W_{2}=\emptyset, \mathcal{M} \vDash_{W_{1}} y=c_{l} \wedge \vec{z}_{1}=\vec{x}_{1} \wedge \vec{z}_{2}=\vec{c}_{1}$ and $\mathcal{M} \vDash_{W_{2}} y=c_{r} \wedge \vec{z}_{1}=\vec{c}_{1} \wedge \vec{z}_{2}=\vec{x}_{2}$.

As above, since $\mathcal{M} \vDash_{X^{\prime}} \theta^{\prime}$, there are $\mathcal{F}_{1}^{\prime}: X^{\prime} \rightarrow M^{k}$ and $\mathcal{F}_{2}^{\prime}: X^{\prime}\left[\mathcal{F}_{1}^{\prime} / \vec{z}_{1}\right] \rightarrow M^{k}$ s.t. $\mathcal{M} \vDash_{Z^{\prime}} \vec{x}_{1} \subseteq \vec{z}_{1}, \mathcal{M} \vDash_{Z^{\prime}} \vec{x}_{2} \subseteq \vec{z}_{2}$, where $Z^{\prime}:=X\left[\mathcal{F}_{1}^{\prime} / \vec{z}_{1}, \mathcal{F}_{2}^{\prime} / \vec{z}_{2}\right]$. Moreover there are subteams $W_{1}^{\prime}, W_{2}^{\prime} \subseteq Z^{\prime}$ such that $W_{1}^{\prime} \cup W_{2}^{\prime}=Z^{\prime}, W_{1}^{\prime} \cap W_{2}^{\prime}=\emptyset$, $\mathcal{M} \vDash_{W_{1}^{\prime}} y=c_{l} \wedge \vec{z}_{1}=\vec{x}_{1} \wedge \vec{z}_{2}=\vec{c}_{2}$ and $\mathcal{M} \vDash_{W_{2}^{\prime}} y=c_{r} \wedge \vec{z}_{1}=\vec{c}_{2} \wedge \vec{z}_{2}=\vec{x}_{2}$. 
Suppose, for the sake of contradiction, that there is $\vec{e} \in X\left(\vec{x}_{1}\right) \cap X\left(\vec{x}_{2}\right)$. Hence there are $s_{1}, s_{2} \in X$ s.t. $s_{1}\left(\vec{x}_{1}\right)=\vec{e}=s_{2}\left(\vec{x}_{2}\right)$. Let $r_{1}:=s_{1}\left[F\left(s_{1}\right) / y\right]$ and $r_{2}:=s_{2}\left[F\left(s_{2}\right) / y\right]$. Now we must have $r_{1}(y), r_{2}(y) \in\{a, b\}$.

Suppose first that $r_{1}(y)=a$ and $r_{2}(y)=b$, whence $r_{1} \in Y$ and $r_{2} \in Y^{\prime}$. Since $\mathcal{M} \vDash_{Y} \vec{x}_{1} \subseteq \vec{z}$, we must have $\vec{e}=r_{1}\left(\vec{x}_{1}\right) \in Y(\vec{z})$. And since $\mathcal{M} \vDash_{Y^{\prime}} \vec{x}_{2} \subseteq \vec{z}$, we must have $\vec{e}=r_{2}\left(\vec{x}_{2}\right) \in Y^{\prime}(\vec{z})$. But this is impossible, since we deduced above that $Y(\vec{z}) \cap Y^{\prime}(\vec{z})=\emptyset$. The case when $r_{1}(y)=b$ and $r_{2}(y)=a$ leads to a contradiction with a symmetric reasoning.

Suppose then that $r_{1}(y)=a=r_{2}(y)$, whence $r_{1}, r_{2} \in Y$. As above, we must have $\vec{e} \in Y(\vec{z})$. Let $r_{2}^{\prime} \in Z$ be the assignment that is obtained by extending $r_{2}$ with $\mathcal{F}_{1}$ and $\mathcal{F}_{2}$. Since $\mathcal{M} \vDash_{Z} \vec{x}_{2} \subseteq \vec{z}_{2}$, there is $r_{3}^{\prime} \in Z$ s.t. $r_{3}^{\prime}\left(\vec{z}_{2}\right)=r_{2}^{\prime}\left(\vec{x}_{2}\right)$. Suppose first that $r_{3}^{\prime} \in W_{2}$, whence $r_{3}^{\prime}(y)=r_{3}^{\prime}\left(c_{r}\right)$ and $r_{3}^{\prime}\left(\vec{z}_{2}\right)=r_{3}^{\prime}\left(\vec{x}_{2}\right)$. Let $r_{3} \in X^{\prime}$ be the assignment that becomes $r_{3}^{\prime}$ when extending it with $\mathcal{F}_{1}$ and $\mathcal{F}_{2}$. Since $r_{3}^{\prime}(y)=r_{3}^{\prime}\left(c_{r}\right)$, also $r_{3}(y)=r_{3}\left(c_{r}\right)$ and thus $r_{3} \in Y^{\prime}$. Now we have $r_{3}\left(\vec{x}_{2}\right)=r_{3}^{\prime}\left(\vec{x}_{2}\right)=r_{3}^{\prime}\left(\vec{z}_{2}\right)=r_{2}^{\prime}\left(\vec{x}_{2}\right)=r_{2}\left(\vec{x}_{2}\right)=s_{2}\left(\vec{x}_{2}\right)=\vec{e}$ and thus $\vec{e} \in Y^{\prime}\left(\vec{x}_{2}\right)$. But since $\mathcal{M} \vDash_{Y^{\prime}} \vec{x}_{2} \subseteq \vec{z}$, we also have $\vec{e} \in Y^{\prime}(\vec{z})$. But this is impossible since $\vec{e} \in Y(\vec{z})$ and we have shown that $Y(\vec{z}) \cap Y^{\prime}(\vec{z})=\emptyset$. Thus $r_{3}^{\prime}$ cannot be in $W_{2}$.

Suppose then that $r_{3}^{\prime} \in W_{1}$, whence $r_{3}^{\prime}\left(\vec{z}_{2}\right)=r_{3}^{\prime}\left(\vec{c}_{1}\right)$ and thus $r_{2}^{\prime}\left(\vec{x}_{2}\right)=\vec{a}$. Let $r_{2}^{\prime \prime} \in Z^{\prime}$ be the assignment that is obtained by extending $r_{2}$ with $\mathcal{F}_{1}^{\prime}$ and $\mathcal{F}_{2}^{\prime}$. Since $\mathcal{M} \vDash_{Z^{\prime}} \vec{x}_{2} \subseteq \vec{z}_{2}$, there is $r_{3}^{\prime \prime} \in Z$ s.t. $r_{3}^{\prime \prime}\left(\vec{z}_{2}\right)=r_{2}^{\prime \prime}\left(\vec{x}_{2}\right)$. If $r_{3}^{\prime \prime} \in W_{2}^{\prime}$, then we obtain a contradiction with a similar reasoning as for $r_{3}^{\prime}$ above. Hence we must have $r_{3}^{\prime \prime} \in W_{1}^{\prime}$. But then $r_{3}^{\prime \prime}\left(\vec{z}_{2}\right)=r_{3}^{\prime}\left(\vec{c}_{2}\right)=\vec{b}$. But this a contradiction since $r_{3}^{\prime \prime}\left(\vec{z}_{2}\right)=r_{2}^{\prime \prime}\left(\vec{x}_{2}\right)=r_{2}\left(\vec{x}_{2}\right)=r_{2}^{\prime}\left(\vec{x}_{2}\right)=\vec{a} \neq \vec{b}$.

The case when $r_{1}(y)=b=r_{2}(y)$ leads to a contradiction with a symmetric reasoning to the previous case. Since all the possible cases lead to a contradiction, we must have $X\left(\vec{x}_{1}\right) \cap X\left(\vec{x}_{2}\right)=\emptyset$.

For the proof of Theorem 5.3, we need also some sufficient conditions for the truth of $\operatorname{exc}_{c_{l}, c_{r}, \vec{z}}\left(\vec{x}_{1}, \vec{x}_{2}\right)$ in a team. The assumptions in the following lemma are very specific as this lemma is formulated particularly for the proof of Theorem 5.3. (The operator $\operatorname{exc}_{c_{l}, c_{r}, \vec{z}}\left(\vec{x}_{1}, \vec{x}_{2}\right)$ is not very interesting in its own right - it is just a tool for our translation from $\operatorname{ESO}[k]$ to $\operatorname{INC}^{s}[k]$.)

Lemma 5.2. Let $\mathcal{M}$ be a model and let $X$ be a team where $c_{l}$ and $c_{r}$ have different constant values $a$ and $b$, respectively. We assume that the following conditions hold for the team $X$.

1. $X\left(\vec{x}_{1}\right) \cap X\left(\vec{x}_{2}\right)=\emptyset$.

2. For each $s \in X$ either $s(\vec{z})=s\left(\vec{x}_{1}\right)$ or $s(\vec{z})=s\left(\vec{x}_{2}\right)$.

3. For each $\vec{a}_{1} \in X\left(\vec{x}_{1}\right)$, there is $s \in X$ for which $s\left(\vec{x}_{1}\right)=\vec{a}_{1}=s(\vec{z})$.

4. For each $\vec{a}_{2} \in X\left(\vec{x}_{2}\right)$, there is $s \in X$ for which $s\left(\vec{x}_{2}\right)=\vec{a}_{2}=s(\vec{z})$.

Now it holds that $\mathcal{M} \vDash_{X} \operatorname{exc}_{c_{l}, c_{r}, \vec{z}}\left(\vec{x}_{1}, \vec{x}_{2}\right)$.

Proof. We first note that by the assumptions 1 and 2, it is impossible that $s\left(\vec{x}_{1}\right)=s(\vec{z})=s\left(\vec{x}_{2}\right)$ for any $s \in X$. Hence, by the assumption 2 , we can define 
the following function:

$$
F: X \rightarrow M, \quad \begin{cases}s \mapsto a & \text { if } s(\vec{z})=s\left(\vec{x}_{1}\right) \\ s \mapsto b & \text { if } s(\vec{z})=s\left(\vec{x}_{2}\right) .\end{cases}
$$

Let $X^{\prime}:=X[F / y], Y:=\{s \in X \mid s(y)=a\}$ and $Y:=\{s \in X \mid s(y)=b\}$. Now clearly $Y, Y^{\prime} \subseteq X^{\prime}, Y \cup Y^{\prime}=X^{\prime}$ and $Y \cap Y^{\prime}=\emptyset$. By the definition of $F$ it is easy to see that $\mathcal{M} \vDash_{Y} y=c_{l} \wedge \vec{x}_{1} \subseteq \vec{z}$ and $\mathcal{M} \vDash_{Y^{\prime}} y=c_{r} \wedge \vec{x}_{2} \subseteq \vec{z}$. Let $\vec{a}$ be the $k$-tuple $\vec{a}:=a \ldots a$ and let

$$
\begin{aligned}
& \mathcal{F}_{1}: X^{\prime} \rightarrow M^{k}, \quad \begin{cases}s \mapsto s\left(\vec{x}_{1}\right) & \text { if } s(y)=a \\
s \mapsto \vec{a} & \text { if } s(y)=b\end{cases} \\
& \mathcal{F}_{2}: X^{\prime}\left[\mathcal{F}_{1} / \vec{z}_{1}\right] \rightarrow M^{k}, \quad \begin{cases}s \mapsto \vec{a} & \text { if } s(y)=a \\
s \mapsto s\left(\vec{x}_{2}\right) & \text { if } s(y)=b .\end{cases}
\end{aligned}
$$

We define the teams $Z:=X\left[\mathcal{F}_{1} / \vec{z}_{1}, \mathcal{F}_{2} / \vec{z}_{2}\right], W_{1}:=\{s \in Z \mid s(y)=a\}$ and $W_{2}:=\{s \in Z \mid s(y)=b\}$. We clearly have $W_{1} \cup W_{2}=Z, W_{1} \cap W_{2}=$ $\emptyset, \mathcal{M} \vDash_{W_{1}} y=c_{l} \wedge \vec{z}_{1}=\vec{x}_{1} \wedge \vec{z}_{2}=\vec{c}_{1}$ and $\mathcal{M} \vDash_{W_{2}} y=c_{r} \wedge \vec{z}_{1}=\vec{c}_{1} \wedge \vec{z}_{2}=\vec{x}_{2}$. For the sake of showing that $\mathcal{M} \vDash_{Z} \vec{x}_{1} \subseteq \vec{z}_{1}$, let $r \in Z$. Let $s \in X$ be the assignment that becomes $r$, when it is extended with $F, \mathcal{F}_{1}$ and $\mathcal{F}_{2}$. By the assumption 3 , there is $s^{\prime} \in X$ such that $s^{\prime}(\vec{z})=s\left(\vec{x}_{1}\right)=s^{\prime}\left(\vec{x}_{1}\right)$. Let then $r^{\prime}:=s^{\prime}\left[a / y, s^{\prime}\left(\vec{x}_{1}\right) / \vec{z}_{1}, \vec{a} / \vec{z}_{2}\right]$. Now $r^{\prime} \in W_{1}$ and $r^{\prime}\left(\vec{z}_{1}\right)=s^{\prime}\left(\vec{x}_{1}\right)=s\left(\vec{x}_{1}\right)=r\left(\vec{x}_{1}\right)$. By using the assumption 4 , we can analogously show that $\mathcal{M} \vDash_{Z} \vec{x}_{2} \subseteq \vec{z}_{2}$ and therefore $\mathcal{M} \vDash_{X^{\prime}} \theta$. Moreover, we can show by a similar reasoning that $\mathcal{M} \vDash_{X^{\prime}} \theta^{\prime}$, which concludes the proof.

\subsection{Translation from $\mathrm{ESO}[k]$ to $\mathrm{INC}^{s}[k]$}

We can formulate a translation from $\operatorname{ESO}[k]$ to $\operatorname{INC}^{s}[k]$ by using very similar ideas as in our translation form $\mathrm{ESO}[k]$ to $\mathrm{EXC}[k]$. As noticed before, we can simulate exclusion atoms with inclusion atoms if we have access to the complementary values in the team: Let $X$ be a team and $\vec{x}, \vec{w}_{i}, \vec{w}_{i}^{c}$ tuples s.t. $X\left(\vec{w}_{i}^{c}\right)=\overline{X\left(\vec{w}_{i}\right)}$. Then we have: $\mathcal{M} \vDash_{X} \vec{x} \mid \vec{w}_{i}$ iff $\mathcal{M} \vDash_{X} \vec{x} \subseteq \vec{w}_{i}^{c}$.

As in the translation in the proof of Theorem 4.5. we use label variables $w_{i}^{\circ}$ and $w_{i}^{\bullet}$ for simulating the quantification of the empty relation and the full relation $M^{k}$. Furthermore, we need again Lemma 4.4 for handling the special case of single element models. One surprising feature of this translation is that we can translate disjunctions directly as $(\psi \vee \theta)^{\prime}=\psi^{\prime} \vee \theta^{\prime}$; this time there is no need for term value preserving disjunction or any other trick as we may allow some of the values of tuples $\vec{w}_{i}, \vec{w}_{i}^{c}$ to be lost when evaluating disjunctions.

The structure of the following proof has many similarities with the proof of Theorem 4.5 and we will omit the parts that can be done here analogously. However, there are also many parts that look similar but which are proven by using different assumptions and thus need to be presented with all the details.

Theorem 5.3. Let $\Phi$ be an $\mathrm{ESO}_{L}[k]$-sentence. Now there exists an $\mathrm{INC}_{L}[k]$ sentence $\varphi$ such that

$$
\mathcal{M} \vDash \varphi \quad \text { iff } \mathcal{M} \vDash \Phi \text {. }
$$


Proof. Since $\Phi$ is an $\mathrm{ESO}_{L}[k]$-sentence, there exists a $\mathrm{FO}_{L}$-sentence $\delta$ and relation symbols $P_{1}, \ldots, P_{n}$ so that $\Phi=\exists P_{1} \ldots \exists P_{n} \delta$. We may assume again that $P_{1}, \ldots, P_{n}$ are all $k$-ary. Let $w_{1}^{\circ}, \ldots, w_{n}^{\circ}, w_{1}^{\bullet}, \ldots, w_{n}^{\bullet}, \vec{w}_{1}, \ldots, \vec{w}_{n}$ and $\vec{w}_{1}^{c}, \ldots, \vec{w}_{n}^{c}$ be as in the proof of Theorem 4.5. Let $u$ and $u^{\prime}$ be fresh variables.

Let $\psi \in \operatorname{Sf}(\delta)$. The formula $\psi^{\prime}$ is defined recursively:

$$
\begin{aligned}
\psi^{\prime} & =\psi, \text { if } \psi \text { is a literal and } P_{i} \text { does not occur in } \psi \text { for any } i \leq n \\
\left(P_{i} \vec{t}\right)^{\prime} & =\left(\vec{t} \subseteq \vec{w}_{i} \vee w_{i}^{\bullet}=u\right) \wedge w_{i}^{\circ} \neq u \quad \text { for all } i \leq n \\
\left(\neg P_{i} \vec{t}\right)^{\prime} & =\left(\vec{t} \subseteq \vec{w}_{i}^{c} \vee w_{i}^{\circ}=u\right) \wedge w_{i}^{\bullet} \neq u \quad \text { for all } i \leq n \\
(\psi \wedge \theta)^{\prime} & =\psi^{\prime} \wedge \theta^{\prime} \\
(\psi \vee \theta)^{\prime} & =\psi^{\prime} \vee \theta^{\prime} \\
(\exists x \psi)^{\prime} & =\exists x \psi^{\prime} \\
(\forall x \psi)^{\prime} & =\forall x \psi^{\prime} .
\end{aligned}
$$

Let $\chi$ be a $\mathrm{FO}_{L}$-sentence determined by the lemma 4.4 for the sentence $\Phi$ and let $\vec{z}$ be a $k$-tuple of fresh variables. We can now define $\varphi$ as follows:

$$
\begin{aligned}
\varphi:=\left(\gamma_{=1} \wedge \chi\right) \vee \exists u \exists u^{\prime}\left(u \neq u^{\prime} \wedge \exists w_{1}^{\circ} \ldots \exists w_{n}^{\circ} \exists w_{1}^{\bullet} \ldots \exists w_{n}^{\bullet}\right. \\
\left.\forall \vec{z} \exists \vec{w}_{1} \ldots \exists \vec{w}_{n} \exists \vec{w}_{1}^{c} \ldots \exists \vec{w}_{n}^{c}\left(\bigwedge_{i=1}^{n} \operatorname{exc}_{u, u^{\prime}, \vec{z}}\left(\vec{w}_{i}, \vec{w}_{i}^{c}\right) \wedge \delta^{\prime}\right)\right) .
\end{aligned}
$$

Clearly now $\varphi$ is an $\operatorname{INC}_{L}[k]$-sentence.

Remark. Since we are using the tuples $\vec{w}_{i}$ and $\vec{w}_{i}^{c}$ to simulate a quantified relation and its complement, it would be natural to require that the union of these values forms the full relation $M^{k}$. This could be achieved by adding the requirement $\bigwedge_{i<n} \forall \vec{v}\left(\vec{v} \subseteq w_{i} \vee \vec{v} \subseteq \vec{w}_{i}^{c}\right)$ to the sentence $\varphi$ above. However, we will see that this is not necessary, since it suffices that $\vec{w}_{i}$ and $\vec{w}_{i}^{c}$ are quantified in such a way that $X\left(\vec{w}_{i}\right) \cap X\left(\vec{w}_{i}^{c}\right)=\emptyset$ in the resulting team..$^{7}$

Before proving the claim of this theorem, we prove the following two claims. The first claim is quite similar to Claim 1. But here instead of assuming that $X\left(\vec{w}_{i}\right) \cup X\left(\vec{w}_{i}^{c}\right)=M^{k}$ we dually assume that $X\left(\vec{w}_{i}\right) \cap X\left(\vec{w}_{i}^{c}\right)=\emptyset$. Also, when defining the sets $A_{i}$, we cannot simply define $A_{i}=X\left(\vec{w}_{i}\right)$ as before. Instead, we must prove that any set $B$ for which $X\left(\vec{w}_{i}\right) \subseteq B \subseteq \overline{X\left(\vec{w}_{i}^{c}\right)}$ could be chosen as $A_{i}$ (this requirement makes sense since $X\left(\vec{w}_{i}\right) \cap X\left(\vec{w}_{i}^{c}\right)=\emptyset$ for each $i \leq n$ ). This strengthening of the claim is crucial for proving the case of disjunction.

We write

$$
V^{*}:=\operatorname{Vr}\left(u u^{\prime} w_{1}^{\circ} \ldots w_{n}^{\circ} w_{1}^{\bullet} \ldots w_{n}^{\bullet} \vec{w}_{1} \ldots \vec{w}_{n} \vec{w}_{1}^{c} \ldots \vec{w}_{n}^{c}\right) .
$$

Claim 3. Let $\mathcal{M}$ be an L-model with at least two elements. Let $\mu \in \operatorname{Sf}(\delta)$ and let $X$ a team for which $V^{*} \subseteq \operatorname{dom}(X)$ and the following assumptions hold:

$$
\left\{\begin{array}{l}
X\left(\vec{w}_{i}\right) \cap X\left(\vec{w}_{i}^{c}\right)=\emptyset \text { for each } i \leq n . \\
\text { The values of } w_{i}^{\circ}, w_{i}^{\bullet}(i \leq n), u \text { and } u^{\prime} \text { are constants in } X .
\end{array}\right.
$$

\footnotetext{
${ }^{7}$ Recall that in the proof of Theorem 4.5 we had to require that $X\left(\vec{w}_{i}\right) \cup X\left(\vec{w}_{i}^{c}\right)=M^{k}$. This difference forms is an interesting piece of duality between these two translations.
} 
We consider functions $H_{X}:\{1, \ldots, n\} \rightarrow \mathcal{P}\left(M^{k}\right)$ s.t. for each $i \leq n$ we have

$$
X\left(\vec{w}_{i}\right) \subseteq H_{X}(i) \subseteq \overline{X\left(\vec{w}_{i}^{c}\right)} .
$$

Let $\mathcal{M}_{H_{X}}:=\mathcal{M}[\vec{A} / \vec{P}]$, where

$$
A_{i}= \begin{cases}\emptyset & \text { if } X\left(w_{i}^{\circ}\right)=X(u) \text { and } X\left(w_{i}^{\bullet}\right) \neq X(u) \\ M^{k} & \text { if } X\left(w_{i}^{\bullet}\right)=X(u) \text { and } X\left(w_{i}^{\circ}\right) \neq X(u) \\ H_{X}(i) & \text { else. }\end{cases}
$$

Now the following implication holds for every function $H_{X}$ :

$$
\text { If } \mathcal{M} \vDash_{X} \mu^{\prime} \text {, then } \mathcal{M}_{H_{X}} \vDash_{X} \mu \text {. }
$$

We prove this claim by structural induction on $\mu$ :

- If $\mu$ is a literal and $P_{i}$ does not occur in $\mu$ for any $i \leq n$, then the claim holds trivially since $\mu^{\prime}=\mu$.

- Let $\mu=P_{j} \vec{t}$ for some $j \leq n$. Suppose that we have $\mathcal{M} \vDash_{X}\left(P_{j} \vec{t}\right)^{\prime}$, i.e. $\mathcal{M} \vDash_{X}\left(\vec{t} \subseteq \vec{w}_{j} \vee w_{j}^{\bullet}=u\right) \wedge w_{j}^{\circ} \neq u$. Because the values of $u, w_{j}^{\circ}$ are constants in $X$ and $\mathcal{M} \vDash_{X} w_{j}^{\circ} \neq u$, we have $X\left(w_{j}^{\circ}\right) \neq X(u)$. If $X\left(w_{j}^{\circ}\right)=X(u)$, then $A_{j}=M^{k}$ and thus trivially $\mathcal{M}_{H_{X}} \vDash_{X} P_{j} \vec{t}$. Suppose then that $X\left(w_{j}^{\bullet}\right) \neq$ $X(u)$ whence $A_{j}=H_{X}(j)$. Because the values of $u, w_{j}^{\bullet}$ are constants in $X$ and $\mathcal{M} \vDash_{X} \vec{t} \subseteq \vec{w}_{j} \vee w_{j}^{\bullet}=u$, it must hold that $\mathcal{M} \vDash_{X} \vec{t} \subseteq \vec{w}_{j}$. Now $X(\vec{t}) \subseteq X\left(\vec{w}_{j}\right) \subseteq H_{X}(j)=A_{j}$ and therefore $\mathcal{M}_{H_{X}} \vDash_{X} P_{j} \vec{t}$.

- Let $\mu=\neg P_{j} \vec{t}$ for some $j \leq n$. Suppose that we have $\mathcal{M} \vDash_{X}\left(\neg P_{j} \vec{t}\right)^{\prime}$, i.e. $\mathcal{M} \vDash_{X}\left(\vec{t} \subseteq \vec{w}_{j}^{c} \vee w_{j}^{\circ}=u\right) \wedge w_{j}^{\bullet} \neq u$. Because the values of $u, w_{j}^{\bullet}$ are constants and $\mathcal{M} \vDash_{X} w_{j}^{\bullet} \neq u$, we have $X\left(w_{j}^{\bullet}\right) \neq X(u)$. If $X\left(w_{j}^{\circ}\right)=X(u)$, then $A_{j}=$ $\emptyset$ and thus trivially $\mathcal{M}_{H_{X}} \vDash_{X} \neg P_{j} \vec{t}$. Suppose then that $X\left(w_{i}^{\circ}\right) \neq X(u)$ whence $A_{j}=H_{X}(j)$. Because the values of $u, w_{j}^{\circ}$ are constants in $X$ and $\mathcal{M} \vDash_{X} \vec{t} \subseteq \vec{w}_{j}^{c} \vee w_{j}^{\circ}=u$, we have $\mathcal{M} \vDash_{X} \vec{t} \subseteq \vec{w}_{j}^{c}$. Because $H_{X}(j) \subseteq \overline{X\left(w_{j}^{c}\right)}$, it also holds that $X\left(\vec{w}_{j}^{c}\right) \subseteq \overline{H_{X}(j)}$. Therefore $X(\vec{t}) \subseteq X\left(\vec{w}_{j}^{c}\right) \subseteq \overline{H_{X}(j)}=\overline{A_{j}}$ and thus $\mathcal{M}_{H_{X}} \vDash_{X} \neg P_{j} \vec{t}$.

- Let $\mu=\psi \vee \theta$. Suppose that $\mathcal{M} \vDash_{X}(\psi \vee \theta)^{\prime}$, i.e. $\mathcal{M} \vDash_{X} \psi^{\prime} \vee \theta^{\prime}$. Hence there are $Y, Y^{\prime} \subseteq X$ s.t. $Y \cup Y^{\prime}=X, Y \cap Y^{\prime}=\emptyset, \mathcal{M} \vDash_{Y} \psi^{\prime}$ and $\mathcal{M} \vDash_{Y^{\prime}} \theta^{\prime}$. Since $X\left(\vec{w}_{i}\right) \cap X\left(\vec{w}_{i}^{c}\right)=\emptyset$ for each $i \leq n$, we must also have $Y\left(\vec{w}_{i}\right) \cap Y\left(\vec{w}_{i}^{c}\right)=$ $\emptyset=Y^{\prime}\left(\vec{w}_{i}\right) \cap Y^{\prime}\left(\vec{w}_{i}^{c}\right)$ for each $i \leq n$. Moreover, since the values of $w_{i}^{\circ}$, $w_{i}^{\bullet}(i \leq n)$ and $u$ are constants in $X$, they must also have (the same) constant values in $Y$ and $Y^{\prime}$.

By the inductive hypothesis, $\mathcal{M}_{H_{Y}} \vDash_{Y} \psi$ and $\mathcal{M}_{H_{Y^{\prime}}} \vDash_{Y} \theta$, for every function $H_{Y}$ and $H_{Y^{\prime}}$. We then consider an arbitrary function $H_{X}$. Since $Y\left(\vec{w}_{i}\right) \subseteq X\left(\vec{w}_{i}\right)$ and $\overline{X\left(\vec{w}_{i}^{c}\right)} \subseteq \overline{Y\left(\vec{w}_{i}^{c}\right)}$ for each $i \leq n$, we have $\mathcal{M}_{H_{X}} \vDash_{Y} \psi$. By a symmetric argumentation $\mathcal{M}_{H_{X}} \vDash_{Y^{\prime}} \theta$. Therefore $\mathcal{M}_{H_{X}} \vDash_{X} \psi \vee \theta$.

- The cases $\mu=\psi \wedge \theta, \mu=\exists x \psi$ and $\mu=\forall x \psi$ are straightforward to prove. 
The next claim is very similar to Claim 2, However, since we cannot use locality nor downward closure properties with $\mathrm{INC}^{s}$, we must prove this claim more generally for an extended team $Z$ which: (1) matches with $X$ when restricted $\operatorname{dom}(X)$; and (2) has the same values as $X^{\prime}$ for certain tuples in $V^{*}$.

Claim 4. Let $\mathcal{M}$ be an L-model with at least two elements. Let $\mu \in \operatorname{Sf}(\delta)$ and $X$ be a team such that $\operatorname{dom}(X)=\operatorname{Fr}(\mu)$. Assume that $A_{1}, \ldots, A_{n} \subseteq M^{k}$, $\mathcal{M}^{\prime}:=\mathcal{M}[\vec{A} / \vec{P}]$ and $a, b \in M$ s.t. $a \neq b$. We write $\vec{a}:=a \ldots a$. Let now

$$
\begin{aligned}
& X^{\prime}:=X\left[\{a\} / u,\{b\} / u^{\prime}, B_{1}^{\circ} / w_{1}^{\circ}, \ldots, B_{n}^{\circ} / w_{n}^{\circ}, B_{1}^{\bullet} / w_{1}^{\bullet}, \ldots, B_{n}^{\bullet} / w_{n}^{\bullet},\right. \\
& \left.B_{1} / \vec{w}_{1}, \ldots, B_{n} / \vec{w}_{n}, B_{1}^{c} / \vec{w}_{1}^{c}, \ldots, B_{n}^{c} / \vec{w}_{n}^{c}\right], \\
& \text { where } \begin{cases}B_{i}^{\circ}=\{a\}, B_{i}^{\bullet}=\{b\}, B_{i}=\{\vec{a}\} \text { and } B_{i}^{c}=M^{k} \backslash\{\vec{a}\} & \text { if } A_{i}=\emptyset \\
B_{i}^{\circ}=\{b\}, B_{i}^{\bullet}=\{a\}, B_{i}=\{\vec{a}\} \text { and } B_{i}^{c}=M^{k} \backslash\{\vec{a}\} & \text { if } A_{i}=M^{k} \\
B_{i}^{\circ}=\{b\}, B_{i}^{\bullet}=\{b\}, B_{i}=A_{i} \text { and } B_{i}^{c}=\overline{A_{i}} & \text { else. }\end{cases}
\end{aligned}
$$

Now the following implication holds:

$$
\text { If } \mathcal{M}^{\prime} \vDash_{X} \mu \text {, then } \mathcal{M} \vDash_{Z} \mu^{\prime} \text {, }
$$

for any team $Z$ for which $Z \uparrow \operatorname{dom}(X)=X$ and $Z(\vec{v})=X^{\prime}(\vec{v})$ for all $\vec{v} \in \vec{V}^{*}$, where

$$
\vec{V}^{*}:=\left\{u, u^{\prime}\right\} \cup \bigcup_{i \leq n}\left\{w_{i}^{\circ}, w_{i}^{\bullet}, \vec{w}_{i}, \vec{w}_{i}^{c}\right\}
$$

We prove this claim by structural induction on $\mu$. If $X=\emptyset$, then also $Z=\emptyset$ and thus the claim holds trivially. Hence we may assume that $X \neq \emptyset$.

- If $\mu$ is a literal and $P_{i}$ does not occur in $\mu$ for any $i \leq n$, then the claim holds by locality (since literals are first order, we may use locality here).

- Let $\mu=P_{j} \vec{t}$ for some $j \leq n$. Suppose that we have $\mathcal{M}^{\prime} \vDash_{X} P_{j} \vec{t}$, i.e. $X(\vec{t}) \subseteq P_{j}^{\mathcal{M}^{\prime}}=A_{j}$. Since $X \neq \emptyset$, also $X(\vec{t}) \neq \emptyset$ and thus $A_{j} \neq \emptyset$. Hence $Z\left(w_{j}^{\circ}\right)=X^{\prime}\left(w_{j}^{\circ}\right)=\{b\}$. Since $Z(u)=X^{\prime}(u)=\{a\}$, we have $\mathcal{M} \vDash_{Z} w_{j}^{\circ} \neq u$. If $A_{j}=M^{k}$, then $Z\left(w_{j}^{\bullet}\right)=X^{\prime}\left(w_{j}^{\bullet}\right)=\{a\}$ and thus $\mathcal{M} \vDash_{Z} w_{j}^{\bullet}=u$. Then $\mathcal{M} \vDash_{Z}\left(\vec{t} \subseteq \vec{w}_{j} \vee w_{j}^{\bullet}=u\right) \wedge w_{j}^{\circ} \neq u$, i.e. $\mathcal{M} \vDash_{Z}\left(P_{j} \vec{t}\right)^{\prime}$. Suppose then that $A_{j} \neq M^{k}$. Now $Z\left(\vec{w}_{j}\right)=X^{\prime}\left(\vec{w}_{j}\right)=A_{j}$ and thus $Z(\vec{t})=X(\vec{t}) \subseteq A_{j}=Z\left(\vec{w}_{j}\right)$. Hence $\mathcal{M} \vDash_{Z} \vec{t} \subseteq \vec{w}_{j}$ and therefore we have $\mathcal{M} \vDash_{Z}\left(\vec{t} \subseteq \vec{w}_{j} \vee w_{j}^{\bullet}=u\right) \wedge w_{j}^{\circ} \neq u$, i.e. $\mathcal{M} \vDash_{Z}\left(P_{j} \vec{t}\right)^{\prime}$.

- Let $\mu=\neg P_{j} \vec{t}$ for some $j \leq n$. Suppose that we have $\mathcal{M}^{\prime} \vDash_{X} \neg P_{j} \vec{t}$, i.e. $X(\vec{t}) \subseteq \overline{P_{j}^{\mathcal{M}^{\prime}}}=\overline{A_{j}}$. Since $X \neq \emptyset$, also $X(\vec{t}) \neq \emptyset$ and thus $\overline{A_{j}} \neq \emptyset$, i.e. $A_{j} \neq M^{k}$. Hence $Z\left(w_{j}^{\bullet}\right)=X^{\prime}\left(w_{j}^{\bullet}\right)=\{b\}$. Since $Z(u)=X^{\prime}(u)=$ $\{a\}$, we have $\mathcal{M} \vDash_{Z} w_{i}^{\bullet} \neq u$. If $A_{j}=\emptyset$, then $Z\left(w_{j}\right)=X^{\prime}\left(w_{j}^{\circ}\right)=\{a\}$ and thus $\mathcal{M} \vDash_{X^{\prime}} w_{j}^{\circ}=u$, whence $\mathcal{M} \vDash_{X^{\prime}}\left(\vec{t} \subseteq \vec{w}_{j}^{c} \vee w_{j}^{\circ}=u\right) \wedge w_{j}^{\bullet} \neq u$, i.e. $\mathcal{M} \vDash_{Z}\left(\neg P_{j} \vec{t}\right)^{\prime}$. Suppose then that $A_{j} \neq \emptyset$. Now $Z\left(\vec{w}_{j}^{c}\right)=X^{\prime}\left(\vec{w}_{j}^{c}\right)=\overline{A_{j}}$ and thus $Z(\vec{t})=X(\vec{t}) \subseteq \overline{A_{j}}=Z\left(\vec{w}_{j}^{c}\right)$. Hence $\mathcal{M} \vDash_{X^{\prime}} \vec{t} \subseteq \vec{w}_{j}^{c}$ and thus $\mathcal{M} \vDash_{X^{\prime}}\left(\vec{t} \subseteq \vec{w}_{j}^{c} \vee w_{j}^{\circ}=u\right) \wedge w_{j}^{\bullet} \neq u$, i.e. $\mathcal{M} \vDash_{X^{\prime}}\left(\neg P_{j} \vec{t}\right)^{\prime}$.

- The case $\mu=\psi \wedge \theta$ is straightforward to prove. 
- Let $\mu=\psi \vee \theta$. Suppose that $\mathcal{M}^{\prime} \vDash_{X} \psi \vee \theta$, i.e. there are $Y_{1}, Y_{2} \subseteq X$ s.t. $Y_{1} \cup Y_{2}=X, Y_{1} \cap Y_{2}=\emptyset, \mathcal{M}^{\prime} \vDash_{Y_{1}} \psi$ and $\mathcal{M}^{\prime} \vDash_{Y_{2}} \theta$. Let $Y_{1}^{\prime}, Y_{2}^{\prime}$ be the teams obtained by extending the teams $Y_{1}, Y_{2}$ as $X^{\prime}$ is obtained by extending $X$. We define the following teams $W_{1}, W_{2} \subseteq Z$ :

$$
\left\{\begin{array}{l}
W_{1}:=\left\{s \in Z \mid s \uparrow \operatorname{dom}(X) \in Y_{1}\right\} \\
W_{2}:=\left\{s \in Z \mid s\left\lceil\operatorname{dom}(X) \in Y_{2}\right\} .\right.
\end{array}\right.
$$

Now $W_{1}\left\lceil\operatorname{dom}\left(Y_{1}\right)=W_{1} \uparrow \operatorname{dom}(X)=Y_{1}\right.$ and $W_{1}(\vec{v})=Y_{1}^{\prime}(\vec{v})$ for all $\vec{v} \in$ $\vec{V}^{*}$. Thus, by the inductive hypothesis, $\mathcal{M} \vDash_{W_{1}} \psi^{\prime}$. By similar reasoning $\mathcal{M} \vDash_{W_{2}} \theta^{\prime}$. It is also easy to see that $W_{1} \cup W_{2}=Z$ and $W_{1} \cap W_{2}=\emptyset$, whence $\mathcal{M} \vDash_{Z} \psi^{\prime} \vee \theta^{\prime}$, i.e. $\mathcal{M} \vDash_{Z}(\psi \vee \theta)^{\prime}$.

- Let $\mu=\exists x \psi$ (the case $\mu=\forall x \psi$ is proven by a similar reasoning). Suppose $\mathcal{M}^{\prime} \vDash_{X} \exists x \psi$, i.e. there is $F: X \rightarrow M$ s.t. $\mathcal{M}^{\prime} \vDash_{W} \psi$, where $W:=X[F / x]$. Let

$$
G: Z \rightarrow M, \quad s \mapsto F(s \uparrow \operatorname{Fr}(\mu))
$$

Note that $G$ is well defined since $\operatorname{dom}(X)=\operatorname{Fr}(\mu)$ and $Z\lceil\operatorname{dom}(X)=X$.

Let $W^{\prime}$ be a team that is obtained by extending the team $W$ analogously as $X^{\prime}$ is obtained by extending $X$. Now by the definition of $G$ we observe that $Z[G / x] \uparrow \operatorname{dom}(W)=W$. Moreover, $(Z[G / x])(\vec{v})=W^{\prime}(\vec{v})$ for all $\vec{v} \in \vec{V}^{*}$. Hence, by the inductive hypothesis, $\mathcal{M} \vDash_{Z[G / x]} \psi^{\prime}$. Therefore $\mathcal{M} \vDash_{Z} \exists x \psi^{\prime}$, i.e. $\mathcal{M} \vDash_{Z}(\exists x \psi)^{\prime}$.

We are now ready to prove the claim of this theorem:

$$
\mathcal{M} \vDash \varphi \text { iff } \mathcal{M} \vDash \Phi \text {. }
$$

Suppose first that $\mathcal{M} \vDash \varphi$. Since the standard disjunction $\vee$ is equivalent with intuitionistic disjunction $\sqcup$ for the singleton team $\{\emptyset\}$, either $\mathcal{M} \vDash \gamma_{=1} \wedge \chi$ or

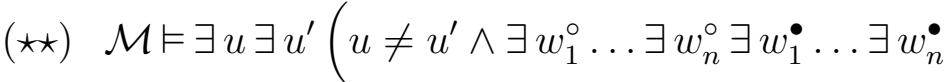

$$
\begin{aligned}
& \left.\forall \vec{z} \exists \vec{w}_{1} \ldots \exists \vec{w}_{n} \exists \vec{w}_{1}^{c} \ldots \exists \vec{w}_{n}^{c}\left(\bigwedge_{i=1}^{n} \operatorname{exc}_{u, u^{\prime}, \vec{z}}\left(\vec{w}_{i}, \vec{w}_{i}^{c}\right) \wedge \delta^{\prime}\right)\right) .
\end{aligned}
$$

If $\mathcal{M} \vDash \gamma_{=1} \wedge \chi$, the claim holds by Lemma 4.4. Suppose then ( $\left.\star \star\right)$, whence by a similar reasoning as in the proof of Theorem 4.5. there exists a team $X_{4}$ such that the following conditions hold 8

- The values of the variables $u, u^{\prime}, w_{i}^{\circ}, w_{i}^{\bullet}(i \leq n)$ are constants in $X_{4}$ and moreover $X_{4}(u) \neq X_{4}\left(u^{\prime}\right)$.

- $\mathcal{M} \vDash_{X_{4}} \bigwedge_{i=1}^{n} \operatorname{exc}_{u, u^{\prime}, \vec{z}}\left(\vec{w}_{i}, \vec{w}_{i}^{c}\right) \wedge \delta^{\prime}$.

\footnotetext{
${ }^{8}$ The team $X_{4}$ here matches the team $X_{4}$ in corresponding part of the proof of Theorem 4.5 with the addition that the variable $u^{\prime}$ is quantified here as a constant that is different from the value of $u$.
} 
We first note that since the variables in $\vec{z}$ were universally quantified and all the other variables in $\operatorname{dom}\left(X_{4}\right)$ were existentially quantified (by the strict semantics), it holds that $\mathcal{M} \vDash_{X_{4}}=(\vec{z}, v)$ for all $v \in \operatorname{dom}(X)$.

Let $j \leq n$. Now the assumptions of Lemma 5.1 hold when $X=X_{4}, c_{l}=u$, $c_{r}=u^{\prime}, \vec{x}_{1}=\vec{w}_{j}$ and $\vec{x}_{2}=\vec{w}_{j}^{c}$. Hence by Lemma $5.1 X_{4}\left(\vec{w}_{j}\right) \cap X_{4}\left(\vec{w}_{j}^{c}\right)=\emptyset$. Now all the assumptions of Claim 3 hold for the team $X_{4}$. Let $\mathcal{M}^{\prime}:=\mathcal{M}[\vec{A} / \vec{P}]$, where

$$
A_{i}= \begin{cases}\emptyset & \text { if } X_{4}\left(w_{i}^{\circ}\right)=X_{4}(u) \text { and } X_{4}\left(w_{i}^{\bullet}\right) \neq X_{4}(u) \\ M^{k} & \text { if } X_{4}\left(w_{i}^{\bullet}\right)=X_{4}(u) \text { and } X_{4}\left(w_{i}^{\circ}\right) \neq X_{4}(u) \\ X_{4}\left(\vec{w}_{i}\right) & \text { else. }\end{cases}
$$

Since $\mathcal{M} \vDash_{X_{4}} \delta^{\prime}$, by Claim 3 we have $\mathcal{M}^{\prime} \vDash_{X_{4}} \delta$ (note that any $A_{i}$ for which $X\left(\vec{w}_{i}\right) \subseteq A_{i} \subseteq \overline{X\left(\vec{w}_{i}^{c}\right)}$, could have been chosen in the last case above). By locality $\left.\right|^{9} \mathcal{M}^{\prime} \vDash \delta$, and therefore $\mathcal{M} \vDash \Phi$.

Suppose then that $\mathcal{M} \vDash \Phi$. If $|M|=1$, then by Lemma 4.4 we have $\mathcal{M} \vDash \gamma_{=1} \wedge \chi$ and thus $\mathcal{M} \vDash \varphi$. Hence we may assume $|M| \geq 2$, whence there are $a, b \in M$ s.t. $a \neq b$. Since $\mathcal{M} \vDash \Phi$, there exist $A_{1}, \ldots, A_{n} \subseteq M^{k}$ s.t. $\mathcal{M}[\vec{A} / \vec{P}] \vDash \delta$. Let

$$
\begin{array}{r}
X^{\prime}:=\{\emptyset\}\left[\{a\} / u,\{b\} / u^{\prime}, B_{1}^{\circ} / w_{1}^{\circ}, \ldots, B_{n}^{\circ} / w_{n}^{\circ}, B_{1}^{\bullet} / w_{1}^{\bullet}, \ldots, B_{n}^{\bullet} / w_{n}^{\bullet},\right. \\
\left.B_{1} / \vec{w}_{1}, \ldots, B_{n} / \vec{w}_{n}, B_{1}^{c} / \vec{w}_{1}^{c}, \ldots, B_{n}^{c} / \vec{w}_{n}^{c}\right],
\end{array}
$$

where $B_{i}^{\circ}, B_{i}^{\bullet}, B_{i}, B_{i}^{c}(i \leq n)$ are defined as in the assumptions of Claim 4 .

Let $\mathcal{F}:\{\emptyset\} \rightarrow M^{2 n+2}$ be the function that gives value $a$ for $u$, value $b$ for $u^{\prime}$ and values for variables $w_{i}^{\bullet}, w_{i}^{\circ}(i \leq n)$ exactly as the corresponding function $\mathcal{F}$ in the proof of Theorem 4.5. Let now $X_{1}:=\{\emptyset\}\left[\mathcal{F} / u u^{\prime} w_{1}^{\circ} \ldots w_{n}^{\circ} w_{1}^{\bullet} \ldots w_{n}^{\bullet}\right]$ and $X_{2}:=X_{1}\left[M^{k} / \vec{z}\right]$. We write $\vec{a}:=a \ldots a$ and fix some $\vec{b}_{i} \in A_{i}$ for each $i \leq n$ for which $A_{i} \neq \emptyset$. We define then the following functions

$$
\mathcal{F}_{i}: X_{2}\left[\mathcal{F}_{1} / \vec{w}_{1}, \ldots, \mathcal{F}_{i-1} / \vec{w}_{i-1}\right] \rightarrow M^{k}, \begin{cases}s \mapsto \vec{a} & \text { if } A_{i}=\emptyset \text { or } A_{i}=M^{k} \\ s \mapsto s(\vec{z}) & \text { if } s(\vec{z}) \in A_{i} \text { and } A_{i} \neq M^{k} \\ s \mapsto \vec{b}_{i} & \text { if } s(\vec{z}) \notin A_{i} \text { and } A_{i} \neq \emptyset\end{cases}
$$

Let $X_{3}:=X_{2}\left[\mathcal{F}_{1} / \vec{w}_{1}, \ldots, \mathcal{F}_{n} / \vec{w}_{n}\right]$. We write $\vec{b}:=b \ldots b$ and fix some $\overrightarrow{b_{i}^{\prime}} \in \overline{A_{i}}$ for each $i \leq n$ for which $A_{i} \neq M^{k}$. Let

$\mathcal{F}_{i}^{\prime}: X_{3}\left[\mathcal{F}_{1}^{\prime} / \vec{w}_{1}^{c}, \ldots, \mathcal{F}_{i-1}^{\prime} / \vec{w}_{i-1}^{c}\right] \rightarrow M^{k},\left\{\begin{array}{l}s \mapsto s(\vec{z}) \text { if } A_{i} \in\left\{\emptyset, M^{k}\right\} \text { and } s(\vec{z}) \neq \vec{a} \\ s \mapsto \vec{b} \text { if } A_{i} \in\left\{\emptyset, M^{k}\right\} \text { and } s(\vec{z})=\vec{a} \\ s \mapsto s(\vec{z}) \text { if } s(\vec{z}) \notin A_{i} \text { and } A_{i} \neq \emptyset \\ s \mapsto \vec{b}_{i}^{\prime} \text { if } s(\vec{z}) \in A_{i} \text { and } A_{i} \neq M^{k} .\end{array}\right.$

Let $X_{4}:=X_{3}\left[\mathcal{F}_{1}^{\prime} / \vec{w}_{1}^{c}, \ldots, \mathcal{F}_{n}^{\prime} / \vec{w}_{n}^{c}\right]$.

Let $j \leq n$. By observing the definitions of $\mathcal{F}_{j}^{\prime}$ and $\mathcal{F}_{j}^{\prime}$, we can see that all the assumptions of Lemma 5.2 hold when $X=X_{4}, c_{l}=u, c_{r}=u^{\prime}, \vec{x}_{1}=\vec{w}_{j}$

\footnotetext{
${ }^{9}$ Note that $\delta$ here is an $\mathrm{FO}_{L}$-sentence and thus locality property may be used.
} 
and $\vec{x}_{2}=\vec{w}_{j}^{c}$. Hence by Lemma 5.2 we have $\mathcal{M} \vDash_{X_{4}} \operatorname{exc}_{u, u^{\prime}, \vec{z}}\left(\vec{w}_{j}, \vec{w}_{j}^{c}\right)$. Moreover, it is easy to see that $X_{4}(\vec{v})=X^{\prime}(\vec{v})$ for every $\vec{v} \in \vec{V}^{*}$ (recall the assumptions of Claim 4. Since $\mathcal{M}[\vec{A} / \vec{P}] \vDash \delta$ and $X_{4}\lceil\operatorname{dom}(\{\emptyset\})=\{\emptyset\}$ by Claim 4 we have $\mathcal{M} \vDash_{X_{4}} \delta^{\prime}$ (note that we cannot use locality property nor downwards closure here as in the proof of Theorem 4.5). Therefore $\mathcal{M} \vDash_{X_{4}} \bigwedge_{i \leq n} \operatorname{exc}_{u, u^{\prime}, \vec{z}}\left(\vec{w}_{i}, \vec{w}_{i}^{c}\right) \wedge \delta^{\prime}$ and moreover $\mathcal{M} \vDash \varphi$.

Corollary 5.4. On the level of sentences $\mathrm{ESO}[k] \leq \mathrm{INC}^{s}[k]$ for any $k \geq 1$.

Thus, by Theorem 4.5, $k$-ary inclusion logic with strict semantics is at least as expressive as $k$-ary exclusion logic on the level of sentences. Recall that by Corollary 4.7 , with the standard (lax) semantics, INC $[k]$ is strictly weaker than $\mathrm{EXC}[k]$ on the level of sentences. Consequently $\mathrm{INC}^{s}[k]$ is strictly more expressive than INC $[k]$ for any $k \geq 1[$.

Since inclusion logic with strict semantics is equivalent with ESO by [6], it would be natural to predict that $\operatorname{INC}^{s}[k] \equiv \operatorname{ESO}[k]$ for any $k \geq 1$. However, for now we only have a lower bound for the expressive power of $\operatorname{INC}^{s}[k]$. To our understanding, a translation from $\mathrm{INC}^{s}[k]$ to $\mathrm{ESO}[k]$ cannot be achieved by modifying the translation from INC $[k]$ to $\mathrm{ESO}[k]$ (in [19]) in any straightforward way. Therefore we leave this question as an open problem for further research.

\subsection{On the relationship between ESO and various logics with team semantics}

We give here some final remarks on the correspondence between ESO and various logics with team semantics. On the level of sentences the whole ESO can be captured with several logics in this framework, such as dependence logic, independence logic, exclusion logic or inclusion logic with strict semantics. But what are the differences between these approaches and which approach can be considered the most natural or practical?

Usually we do not need the whole ESO and some of its simpler fragments suffice. By restricting the arities of atoms in either dependence or independence logic, we can naturally capture all the functional arity fragments of ESO. However, supposing that the functional arity fragments of ESO differ from the relational ones, then the arity fragments of dependence or independence logic cannot capture any of the relational fragments of ESO - of which ESO[1] and ESO $[2]$ are particularly natural. These and all the other relational fragments can be captured with the fragments of INEX and EXC.

By examining the actual translations that have been presented, we believe that the compositional translation from $\operatorname{ESO}[k]$ to INEX $[k]$, presented in [19], is currently the most simple and straightforward. By the results of this paper, we know that inclusion atoms are not needed in order to formulate this translation. However, in order to get rid of inclusion atoms, we had to do several "tricks" which made the translation more complicated and unnatural.

So far we have only considered this correspondence on the level of sentences. In order to capture ESO on the level of formulas, we need either independence

\footnotetext{
${ }^{10}$ For the general case this is a known results by [6]. But, to our best knowledge, this is a new result for bounded arity fragments of inclusion logic.
} 
logic or inclusion-exclusion logic. From the know translations, the one between ESO and INEX ([19]) respects the arity fragments in a natural way. It should also be noted that inclusion atoms are crucial for this translation and we cannot simulate them with exclusion atoms (as we could do on the level of sentences). By the observations given here, we argue the results of this paper rather complement the results of [19] instead of trivializing them.

\section{Conclusion}

In this paper we have analyzed the expressive power of $k$-ary exclusion atoms. We first observed that the expressive power of $\mathrm{EXC}[k]$ is between $k$-ary and $(k+1)$-ary dependence logics, and that when $k=1$, these inclusions are proper. By simulating the use of inclusion atoms with exclusion atoms and by using the complementary values, we were able to translate $\mathrm{ESO}[k]$-sentences into $\mathrm{EXC}[k]$. By combining this with our earlier translation we managed to capture the $k$ ary fragment of ESO by using only $k$-ary exclusion atoms, which resolves the expressive power of $\mathrm{EXC}[k]$ on the level of sentences. However, on the level of formulas our results are not yet conclusive.

As mentioned in the introduction, by [3], on the level of sentences $k$-ary dependence logic captures the fragment of ESO where $(k-1)$-ary functions can be quantified. Thus 1-ary dependence logic is not more expressive than FO, but 2-ary dependence logic is strictly stronger than EMSO - which can be captured with $\operatorname{EXC}[1]$. Also, the question whether $\operatorname{EXC}[k]$ is properly in between $k$-and $(k+1)$-ary dependence logic for all $k \geq 2$, amounts to showing whether $k$-ary relational fragment of ESO is properly between $(k-1)$-ary and $k$-ary functional fragments of ESO for any $k \geq 2$. To our best knowledge this is still an open problem, even though, by the result of Ajtai [1, both relational and functional fragments of ESO have a strict arity hierarchy (over arbitrary vocabulary).

In order to formulate the translation in our main theorem, we needed use a new operator to called unifier which is expressible in exclusion logic. This is a very simple but interesting operator for the framework of team semantics by its own right, and its properties deserve to be studied further - either independently or by adding it to some other logics in this framework.

Finally we used the techniques developed in this paper to formulate a translation from $\mathrm{ESO}[k]$ to $k$-ary inclusion logic with strict semantics $\left(\operatorname{INC}^{s}[k]\right)$. We left as an open problem whether $\operatorname{INC}^{s}[k]$ captures $\mathrm{ESO}[k]$ or is even stronger.

\section{References}

[1] M. Ajtai. $\Sigma_{1}^{1}$-formulae on finite structures. Ann. Pure Appl. Logic, 24:1-48, 1983.

[2] A. Dawar. A restricted second order logic for finite structures. Inf. Comput., 143(2):154-174, 1998.

[3] A. Durand and J. Kontinen. Hierarchies in dependence logic. ACM Trans. Comput. Log., 13(4):31:1-31:21, 2012. 
[4] P. Galliani. The Dynamics of Imperfect Information. ILLC Dissertation Series. Amsterdam: Institute for Logic, Language and Computation, 2012.

[5] P. Galliani. Inclusion and exclusion dependencies in team semantics - on some logics of imperfect information. Ann. Pure Appl. Logic, 163(1):68-84, 2012.

[6] P. Galliani, M. Hannula, and J. Kontinen. Hierarchies in independence logic. In proceedings of CSL 2013, pages 263-280, 2013.

[7] P. Galliani and L. Hella. Inclusion logic and fixed point logic. In proceedings of CSL 2013, pages 281-295, 2013.

[8] E. Grädel and J. A. Väänänen. Dependence and independence. Studia Logica, 101(2):399-410, 2013.

[9] M. Hannula. Hierarchies in inclusion logic with lax semantics. In proceedings of ICLA 2015, pages 100-118, 2015.

[10] M. Hannula and J. Kontinen. Hierarchies in independence and inclusion logic with strict semantics. J. Log. Comput., 25(3):879-897, 2015.

[11] L. Hella, A. Kuusisto, A. Meier, and J. Virtema. Model checking and validity in propositional and modal inclusion logics. In proceedings of MFCS 2017, pages $32: 1-32: 14,2017$.

[12] J. Hintikka and G. Sandu. Informational independence as a semantical phenomenon. In J. E. Fenstad, editor, Logic, Methodology and Philosophy of Science VIII, pages 571-589. North-Holland, 1989.

[13] J. Hintikka and G. Sandu. Game-theoretical semantics. In J. van Benthem and A. ter Meulen, editors, Handbook of Logic and Language, pages 361-410. Elsevier, 1997.

[14] W. Hodges. Compositional semantics for a language of imperfect information. Logic Journal of the IGPL, 5(4):539-563, 1997.

[15] T. Hyttinen, G. Paolini, and J. Väänänen. Quantum team logic and Bell's inequalities. Rew. Symb. Logic, 8:722-742, 122015.

[16] J. Kontinen, S. Link, and J. A. Väänänen. Independence in database relations. In proceedings of WoLLIC 2013, pages 179-193, 2013.

[17] J. Kontinen and J. A. Väänänen. On definability in dependence logic. Journal of Logic, Language and Information, 18(3):317-332, 2009.

[18] R. Rönnholm. The expressive power of $k$-ary exclusion logic. In proceedings of WoLLIC 2016, pages 375-391, 2016.

[19] R. Rönnholm. Capturing $k$-ary existential second order logic with $k$-ary inclusion-exclusion logic. Ann. Pure Appl. Logic, 169(3):177-215, 2018.

[20] J. A. Väänänen. Dependence Logic - A New Approach to Independence Friendly Logic, volume 70 of London Mathematical Society student texts. Cambridge University Press, 2007. 